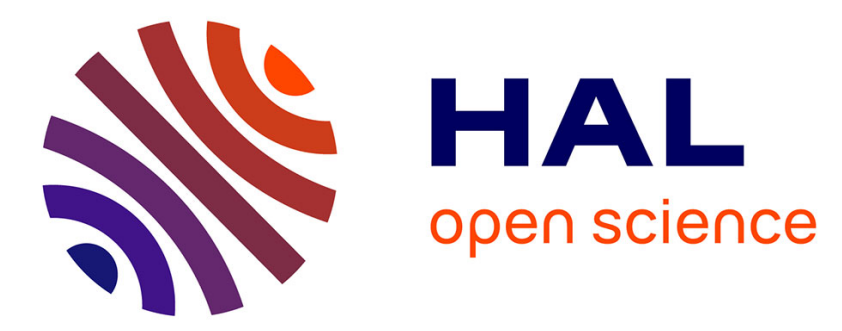

\title{
Zero-field splitting in transition metal complexes: Ab initio calculations, effective Hamiltonians, model Hamiltonians, and crystal-field models
}

\author{
Rémi Maurice, R. Broer, Nathalie Guihéry, C. de Graaf
}

\section{To cite this version:}

Rémi Maurice, R. Broer, Nathalie Guihéry, C. de Graaf. Zero-field splitting in transition metal complexes: Ab initio calculations, effective Hamiltonians, model Hamiltonians, and crystal-field models. Hanbook of Relativistic Quantum Chemistry, 2016, 978-3-642-41611-8. 10.1007/978-3-642-416118_37-1. in2p3-01323359

\section{HAL Id: in2p3-01323359 \\ https://hal.in2p3.fr/in2p3-01323359}

Submitted on 2 Dec 2021

HAL is a multi-disciplinary open access archive for the deposit and dissemination of scientific research documents, whether they are published or not. The documents may come from teaching and research institutions in France or abroad, or from public or private research centers.
L'archive ouverte pluridisciplinaire $\mathbf{H A L}$, est destinée au dépôt et à la diffusion de documents scientifiques de niveau recherche, publiés ou non, émanant des établissements d'enseignement et de recherche français ou étrangers, des laboratoires publics ou privés. 


\section{Zero-field splitting in transition metal complexes: $\boldsymbol{A b}$ initio calculations, effective}

Hamiltonians, model Hamiltonians, and crystal-field models

Rémi Maurice $^{a}$, Ria Broer ${ }^{b}$, Nathalie Guihéryc ${ }^{c}$, and Coen de Graaf ${ }^{b, d}$

a SUBATECH, UMR CNRS 6457, IN2P3/EMN Nantes/Université de Nantes, 4 rue A. Kastler, BP 20722, 44307 Nantes Cedex 3, France. Email: remi.maurice@subatech.in2p3.fr

$b$ Zernike Institute for Advanced Materials, University of Groningen, Nijenborgh 4, Groningen 9747 AG, The Netherlands.Email: r.broer@rug.nl

c Laboratoire de Chimie et Physique Quantiques, IRSAMC/UMR5626, Université de Toulouse 3, 118 route de Narbonne, F-31062 Toulouse Cedex4, France. Email: nathalie.guihery@irsamc.ups-tlse.fr

d Departament de Química Física I Inorgànica, Universitat Rovira i Virgili, Marcel·lí Domingo s/n, 43007 Tarragona, Spain, and Institució Catalana de Recerca i Estudis Avançats (ICREA), Passeig Lluís Companys 23, 08010 Barcelona, Spain. Email: coen.degraaf@urv.cat

\section{Introduction}

Zero-field splitting (ZFS) is a concept that is commonly used to indicate the loss of the degeneracy of the spin components of a spin-orbit free (SOF) state in the absence of an external magnetic field. This picture of ZFS is based on the separation of SOF and spindependent effects such as spin-orbit coupling (SOC). With this viewpoint, ZFS between the spin components of $S>1 / 2$ SOF states can be observed in mononuclear complexes (i.e. complexes with one transition metal -TM- centre), when (i) the "crystal" field felt by the TM ion is anisotropic (i.e. does not correspond to a spherical or cubic symmetry), and (ii) when SOC is sufficiently large [McWeeny, 1965]. Spin-spin coupling (SSC) is also at play [McWeeny and Mizuno, 1961], but usually to a lesser extent. ZFS can in principle occur in any type of systems, however, it is commonly associated with an orbitally nondegenerate ground SOF state. This chapter focuses on TM complexes, and more particularly the $3 d$ ones. Mononuclear and binuclear (i.e. with two TM centres) 
complexes will be considered to introduce the main features of single-ion and molecular or intersite anisotropies.

Although ZFS is a concept that has been known for almost a century, it has triggered new investigations in the last twenty years, among which theoretical studies play a key role. One of the reasons for this revival is the discovery of the single-molecule magnet (SMM) behaviour [Gatteschi and Sessoli, 2003], which was first evidenced in the so-called $\mathrm{Mn}_{12}$ molecule [Caneschi et al., 1991]. In this system, the low-temperature magnetic behaviour is interpreted as coming from the splitting of the spin-components of the $S=10$ SOF ground state. The slow relaxation of the magnetization from the $M_{S}=-10$ to $M_{S}=10$ components and vice-versa, is observable at reasonable experimental timescales. The relaxation occurs via different mechanisms, such as thermal activation, direct tunnelling, or thermally assisted tunnelling. More information concerning SMMs can be for instance found in the nice review of Gatteschi and Sessoli [Gatteschi and Sessoli, 2003]. Let us just recall that in order to design higher-temperature SMMs, various strategies have been attempted, as for instance enlarging the number of TM centres, or enlarging the single-ion anisotropies. More particularly for the latter strategy, it became clear that more extensive studies were necessary to understand the properties of "exotic" coordination spheres with pentacoordinated or heptacoordinated metal centres [Rebilly et al., 2008; Costes et al., 2012; Ruamps et al., 2013a; Ruamps et al., 2013b], or even low-coordinated cases [Zadrozny et al., 2013; Atanasov et al. 2013]. Moreover, the search for magneto-structural correlations from $a b$ initio calculations and crystal-field models appeared necessary to eventually guide the synthesis of new coordination complexes, as highlighted by Telser in 2006 [Telser, 2006].

ZFS also plays an important role in the magnetic properties of condensed matter, as for instance ionic solids. In these systems, TM centres may be the subjects of local and intersite anisotropies. Low-temperature magnetic properties of extended systems are at least partly driven by the microscopic interactions that lead to ZFS. One may quote magnetic multiferroics [Ederer and Spaldin, 2004], for which magnetic and for instance electric transitions are coupled, meaning that one can in principle influence the magnetism of the material by the application of an external electric field or the electric polarization by a magnetic field. One common way of tackling the properties of solids is to treat infinite systems by applying periodic boundary conditions (PBCs), i.e. neglecting edge effects, and assuming an ideally ordered arrangement of the atoms. However, for reasons that 
will be discussed later in this chapter, it is practically impossible to accurately compute ZFS when PBCs are considered. Another approach, which is perfectly suited for the computation of local effects in ionic solids, consists of using an embedded cluster to model the material, as was done by Pradipto et al. to study cupric oxide [Pradipto et al., 2012] and Maurice et al. for $\mathrm{LiCu}_{2} \mathrm{O}_{2}$ [Maurice et al., 2012]. The interested reader may consult more literature concerning this approach [de P. R. Moreira et al., 1999; de P. R. Moreira and Illas, 2006 -and references therein-], but we already stress that the methods and conclusions that are given in this chapter are directly applicable to solids provided that an embedded cluster approach is followed.

ZFS is often described in terms of model Hamiltonians, which have been (almost) always introduced phenomenologically. Such models are typically spin Hamiltonians, since by definition ZFS applies to systems for which the ground orbital configuration is separated in energy from the others, and hence, the effective description of the lowest-lying states can be restricted to the spin variables. For mononuclear complexes, these Hamiltonians only consider the spin anisotropy of the magnetic centre. For polynuclear complexes, two main types of models are widely used, namely the giant-spin and the multispin models. Both types will be discussed here for binuclear systems to keep the discussion as clear as possible.

This chapter aims at (i) making a bridge between $a b$ initio calculations and model Hamiltonians to validate phenomenological Hamiltonians, (ii) establishing magnetostructural correlations, and (iii) demonstrating that magneto-structural correlations can also be understood in terms of crystal-field models. Therefore, in principle, one can bridge ab initio calculations, model Hamiltonians, and crystal-field models to get a full and intuitive picture of the ZFS in TM complexes. The chapter is organized as follows; first, we describe the $a b$ initio methodologies that are sufficiently accurate to compute ZFS, as well as the effective Hamiltonian theory; second, we discuss the relevance of standard and improved model Hamiltonians that effectively describe the ZFS in mononuclear and binuclear complexes; third, we evaluate magneto-structural correlations, either derived from ab initio calculations performed on model complexes, or analysed within crystal-field model; finally, we conclude on the overall progress made in the last two decades, and also give some perspectives.

\section{I. $A b$ initio calculations and effective Hamiltonians}


In this part, we describe a common strategy to introduce relativistic effects in the calculation of ZFS, the typical way to introduce electron correlation in contracted spinorbit configuration interaction (C-SOCI), and a way to bridge the resulting spin-orbit wave functions to model Hamiltonians to (i) assess the validity of model Hamiltonians and (ii) extract the model parameter values if appropriate. Apart from the herediscussed approach, other methods have been developed to compute ZFS and extract the model parameter values [Neese and Solomon, 1998, Pederson and Khanna, 1999; Aquino and Rodriguez, 2005; Neese, 2006; Ganyushin and Neese, 2006; Neese, 2007; Schmitt et al., 2011; Chibotaru and Ungur, 2012], but these are out of the scope of this chapter.

\section{I.1. From the Dirac equation to contracted spin-orbit configuration interaction}

The time-independent Dirac equation may be written as:

$$
\widehat{H} \psi=\left(c \hat{p} \hat{\alpha}+m_{e} c^{2} \hat{\beta}+\widehat{V}\right) \psi=E \psi
$$

where $c$ is the speed of light, $\hat{p}$ is the momentum operator, $\hat{\alpha}$ and $\hat{\beta}$ are the Dirac $4 \times 4$ matrices, $\widehat{V}$ is the one-electron external potential, and $\psi$ is a four-component $(4 c)$ wave function. The resolution of the Dirac equation leads to two different types of solutions, the upper energy eigenfunctions are usually referred to as the "large" components, and the lower energy ones are known as the "small" components. Since the large components tend to standard spin-orbitals in the non-relativistic limit, these may also be considered as "essentially electronic" solutions. Although the introduction of relativistic effects is in principle more natural in $4 c$ frameworks, much effort has been devoted in the last decades to reduce the complexity and derive accurate twocomponent $(2 c)$ models.

Various transformations/approximations have been implemented in standard codes, among which we quote the Douglas-Kroll (DK) transformation based expansions [Douglas and Kroll, 1976; Hess, 1986; Jansen and Hess, 1989] and the zeroth-order regular approximation (ZORA) approach [van Lenthe et al., 1994; van Lenthe et al., 1996]. More recently, exact $2 c$ formalisms (X2C) have been proposed [Iliaš and Saue, 
2007]. Since many investigations of ZFS in TM complexes make use of the DK transformation, we will here describe briefly how to reach c-SOCI formalisms after this transformation, although c-SOCI schemes may also be derived from other reference $2 c$ Hamiltonians.

The DK transformation can in principle lead to the exact energies of the Dirac Hamiltonian if one considers an infinite-order expansion of the one-electron external potential $(\widehat{V})$. In practice, the expansion is of course limited to finite order; in most cases the expansion only contains second order terms. After the sign correction by Jansen and Hess in the original derivation of the transformation [Jansen and Hess, 1989], the second-order (and higher) DK expansions are commonly referred to as the DouglasKroll-Hess (DKHn) Hamiltonians, where $n$ is the expansion order. Another approximation, called the no-pair transformation, can be then introduced, in which the one-electron kinetic Hamiltonian matrix is diagonalised within a (finite) basis set to form a conventional one-electron basis. Although one can in principle use transformed one-electron and two-electron interactions, a one-component (1c) pseudo-relativistic Hamiltonian can be used in scalar relativistic calculations if only the spin-independent one-electron interactions are transformed [Roos and Malmqvist, 2004], which means that non-relativistic two-electron interactions are formally considered. A standard approximation consists of introducing the SOC after a non-relativistic or scalarrelativistic calculation. Assuming that a set of scalar relativistic reference states has been built in the first step, a c-SOCI calculation consists of diagonalizing $\widehat{H}=E_{e l}+\widehat{H}_{S O C}$ within the basis of the spin components of these reference SOF states, in which $E_{e l}$ are SOF electronic energies, and $\widehat{H}_{S O C}$ is an appropriate SOC Hamiltonian. More details concerning the choice of the SOF electronic energies will be given in the next section. Let us just mention that it is common practice nowadays to use electronic energies coming from a higher level of theory than the one that is used to compute the multireference wave functions. So in addition to using the reference wave functions for the off-diagonal SOC matrix elements of $\widehat{H}$, one can "dress" the diagonal elements of a c-SOCI matrix with higher-level electronic energies. Such an approach was proposed by Teichteil et al. [Teichteil et al., 1983] and also Llusar et al. [Llusar et al., 1996].

Due to the local character of the spin-orbit operator, one may neglect interatomic SOCs. Expressions for the atomic SOC Hamiltonian that include one-electron and two-electron interactions adapted to the no-pair DKH2 Hamiltonian can be found elsewhere [Roos 
and Malmqvist, 2004]. If one further applies a mean-field approximation to treat the two-electron part of the atomic SOC Hamiltonian by assuming an atomic one-electron density and adding the resulting mean-fields to the one-electron integrals, the so-called atomic mean-field integral (AMFI) approximation is used [Hess et al., 1996]. Note that alternative mean-field approximations of the SOC operator have been proposed by Neese [Neese, 2005] and that the atomic approximation can nowadays be avoided even in routine calculations.

\section{I.2. The treatment of the spin-orbit free electron correlation}

Although c-SOCI calculations can in principle be performed using single-reference SOF states, this is not an optimal approach to compute ZFS since it is in most cases impossible to converge enough excited SOF states to obtain a good representation of the SOC operator. Therefore, it is more appropriate to use multireference SOF states, which are obtained from multiconfigurational self-consistent field (MCSCF) approaches. A second requirement to compute ZFS is that all the spin components of the SOF state(s) of interest have to be coupled to excited components in a balanced way, i.e. one should not introduce a bias in the treatment of the lowest-energy spin-orbit states towards one specific spin-orbit component. This common-sense requirement has two implications in practice, (i) the set of SOF states considered in the first step of the calculation must be rather well thought, since selections based on an energy basis do not always lead to a consistent choice and (ii) it is convenient to consider state-averaged (SA) orbitals between all the SOF states of interest to control the balance between the SOC excitations that are introduced a posteriori. Although SA orbitals can be calculated for any type of MCSCF multireference SOF states, the appropriate calculation of physical properties requires ensuring some properties of the wave functions, which may break down by any space truncation of the CI space that is used for computing the SOF states. This is why most researchers consider the complete active space self-consistent field (CASSCF) method [Roos et al., 1980] in the first step of a ZFS calculation.

Perhaps it is necessary at this stage to introduce the example of the nearly octahedral $d^{8}$ complexes to illustrate how one can consistently choose balanced active spaces and balanced SOCI spaces, i.e. an adequate set of SOF states in the first step of the calculation. If one only considers the $d^{8}$ manifold, 10 spin-triplet (10T) and 15 spin-singlet (15S) SOF 
states can be at maximum considered. Therefore, the easiest way to define balanced spaces consists of choosing an active space of 8 electrons in 5 orbitals, i.e. a CAS(8/5), and in computing $10 \mathrm{~T}$ and $15 \mathrm{~S}$ SOF states with the state-averaged CASSCF (SA-CASSCF) method [Roos, 2005]. Note that it is easier to guess which active spaces and which sets of states can be suitable by looking at the reference, most symmetrical situation, i.e. in the octahedral case. One can for instance think of adding the two orbitals associated to the ligand-to-metal " $\sigma$ " donation, or to consider the ground SOF state plus a number of excited states that are consistent with the orbital degeneracies in that symmetry point group ("roots"), leading for instance to 4T, 7T and 2S, 10T and 9S sets of SOF states [Maurice et al., 2009]. In any case, the degeneracy of the first three spin-orbit roots should be strictly maintained in the octahedral situation. When the symmetry is lowered, which is necessary to observe ZFS, one should maintain a balanced treatment of the SOC operator. While it is clear that the active spaces that are consistent for the octahedral situation are transferable to the case of nearly octahedral complexes, the definition of the set of SOF states may be problematic. One can always recommend using the full set of states that can be formed within an 8/5 active space (i.e. 10T and 15S), or check that any of the previously mentioned subsets of it leads to similar averaged occupation numbers for the orbitals from which excitations are formed, i.e. for the three orbitals that correspond to the $t_{2 g}$ orbitals in the octahedral case. A similar reasoning can be applied to any $d^{\mathrm{n}}$ configuration near an ideal geometry that leads to orbitally nondegenerate ground states, as for instance in nearly tetrahedral $d^{7}$ complexes. In this case, the "full" set of spin-orbit free states consists of 10 spin-quartet (10Q) and 40 spindoublet (40D) SOF states, while it is also safe to consider the $4 Q$ and $7 Q$ subsets [Maurice et al., 2009].

Now that the set of SOF states has been defined, the SOC is computed between the spin components (i.e. $M_{S}$ components) of these states to form the c-SOCI matrix. However, one needs to further discuss the choice of the SOF electronic energies that appear on the diagonal of the matrix. Since the spin components of the SA-CASSCF SOF states are considered to compute the off-diagonal elements of the c-SOCI matrix, it may appear natural to just consider the SA-CASSCF electronic energies on the diagonal of this matrix. This straightforward choice may however not be the wisest. In mononuclear complexes satisfactory results may be obtained at this level, although it is also clear that results are in general slightly improved when the diagonal of the c-SOCI matrix is "dressed" with 
post-CASSCF correlated energies [Maurice et al., 2009]. In binuclear complexes, ZFS can be severely underestimated if SA-CASSCF energies are considered on the diagonal elements of the c-SOCI matrix, as shown by Maurice et al. showed in a very detailed study concerning the ZFS of the first excited spin-triplet block in copper acetate monohydrate [Maurice et al., 2011a]. In this case, it was shown via a crystal-field model that the ZFS of interest relates at second order of perturbation to the isotropic magnetic couplings of the orbitally single-excited states. As shown by decades of experience, a proper description of isotropic magnetic couplings is not trivial at post-CASSCF levels [Malrieu et al., 2014]. Perturbative approaches such as the complete active space perturbation theory at second order (CASPT2) [Anderson et al., 1992] and the nelectron valence state perturbation theory at second order (NEVPT2) [Angeli et al., 2001] do not fully account for the effect of charge-transfer configurations on the isotropic couplings. Although the description is clearly improved compared to CASSCF, multireference configuration interaction (MRCI) has to be considered for quantitative results. Among the different MRCI schemes, the difference dedicated configuration interaction (DDCI) methods [Miralles et al., 1993] is one of the most successful ones for computing isotropic couplings. This method considers all the single and double excitations minus the double excitations that create two holes $(h)$ in the inactive orbitals and two particles $(p)$ in the virtual orbitals, usually referred to as the $2 h 2 p$ excitations. Numerical examples will be given in sections II.1 and II.2 for mononuclear and binuclear complexes respectively and the comparison with experimental data will be discussed.

\section{I.3. On the effective Hamiltonian theory}

The effective Hamiltonian theory [Bloch, 1958; des Cloizeaux, 1960] enables one to establish a bi-univocal relation between a sophisticated Hamiltonian (here called the "reference" Hamiltonian) expressed in a large reference space and an effective Hamiltonian working on a truncated space, usually of much smaller dimension, that is called the "model" space. By definition, the eigenfunctions of an effective Hamiltonian are the wavefunctions of the reference Hamiltonian projected onto the model space while its eigenvalues are set to be identical to the energies of the reference Hamiltonian. Note that effective Hamiltonians can be used to design computational approaches [Spiegelmann and Malrieu, 1984a; Spiegelmann and Malrieu, 1984b; Chang et al., 1986, 
Finley et al., 1998]. Alternatively, effective Hamiltonians can be used to extract information from wave functions and energies in order to determine the interactions of model Hamiltonians (i.e. the model operators and parameters).. By doing so, computational chemistry can (i) assess the validity of the considered model Hamiltonian by checking that the model space is appropriate (the norms of the projections onto the model space must be large enough) and check that the operators of the model Hamiltonian are the relevant ones (a good one-to-one correspondence between the model Hamiltonian and the effective Hamiltonian matrices must be obtained), and (ii) extract the model parameter values. This approach proved to be particularly appropriate in the field of ZFS for the second purpose, even if alternatives exist, as for instance the pseudo-spin approach of Chiboratu and Ungur [Chibotaru and Ungur, 2012].

Various formulations of effective Hamiltonians have been reported in the literature, among which the Bloch [Bloch, 1956] and des Cloizeaux [des Cloizeaux, 1960] ones that will be commented here. The Bloch formulation of the effective Hamiltonian is defined as:

$$
\widehat{H}_{\text {Bloch }}=\sum_{k}\left|\tilde{\psi}_{k}\right\rangle E_{k}\left\langle S^{-1} \tilde{\psi}_{k}\right|
$$

where $\tilde{\psi}_{k}$ and $E_{k}$ are the $k^{\text {th }}$ projected eigenvector and eigenvalue of the reference Hamiltonian. The projected eigenvectors of the reference Hamiltonian are mutually nonorthogonal and $S^{-1}$ is the inverse of the overlap matrix between the projected eigenvectors. However, one should note that this formulation does not ensure hermiticity of the effective Hamiltonian, which may be problematic for extracting model parameter values. In order to get Hermitian model Hamiltonians the des Cloizeaux formalism, which symmetrically orthogonalizes the projected eigenvectors (usually referred to as the Löwdin's orthogonalization in quantum chemistry), can be used. In this formalism, the effective Hamiltonian is defined as:

$$
\widehat{H}_{\text {des Cloizeaux }}=\sum_{k}\left|S^{-\frac{1}{2}} \tilde{\psi}_{k}\right\rangle E_{k}\left\langle S^{-\frac{1}{2}} \tilde{\psi}_{k}\right|
$$


Examples of discussions on the validity of model Hamiltonians and extractions of model parameter values are given in the next section.

\section{Model Hamiltonians and effective Hamiltonians}

Model Hamiltonians not only reduces the complexity of a given reference Hamiltonian, the model space is always smaller than the reference space, but also introduces effective parameters with a well-defined physical meaning. In the field of molecular magnetism, the typical simpler Hamiltonians are the well-known phenomenological spin Hamiltonians, in which no track of the orbital part of the wave functions is kept. Although the earliest spin Hamiltonian is the Heisenberg-Dirac-van Vleck (HDV) [Heisenberg, 1928; Dirac, 1929; Van Vleck, 1945] one (vide infra), the expression "spin Hamiltonian" was introduced later by Abragam and Pryce [Abragam and Pryce, 1951] in the context of the electron paramagnetic resonance (EPR) spectroscopy. In this part, we will show that (i) the effective Hamiltonian theory can be used to project the information contained in c-SOCI wave functions onto a model space consisting of the spin components of one (or several) spin state(s), (ii) question the validity of model Hamiltonians and improve them if necessary, and (iii) show that model parameter values in good agreement with experiment can be obtained. We will start by discussing mononuclear complexes prior to dealing with binuclear systems to gradually increase the complexity of the models to be introduced. Note that a basic introduction to the use of effective Hamiltonian theory in relation to model Hamiltonians can be found in a recent textbook [de Graaf and Broer, 2015].

\section{II.1. Mononuclear complexes}

Mononuclear complexes with a $d^{\text {n }}$ electronic configuration can be split in two groups, $S=1$ or $S=3 / 2$ systems and $S=2$ or $S=5 / 2$ systems, as the model Hamiltonians that have to be used to describe the full complexity of their ZFS are different. Note that, in a first approximation, the model Hamiltonian for $S=1$ or $S=3 / 2$ systems can be used to describe $S=2$ or $S=5 / 2$ systems. Although this is commonly done in the literature, this approximation may not always be adequate, as will be discussed later. One should also 
stress that usually, in the $S=2$ or $S=5 / 2$ systems, the SSC can contribute to a significant part of the total ZFS, around 10\% of the total ZFS in manganese(III) complexes [Duboc et al., 2010], and even up to $20 \%$ in manganese(II) complexes [Zein and Neese, 2008]. Therefore, one should account for the SSC in the determination of ZFS parameters [Neese, 2006]. A remark is thus worth here. Both SOC and SSC generate second-rank ZFS tensors and, unless these axes are imposed by symmetry, both effects may independently generate different magnetic axes. Therefore, one should in principle not only perform a c-SOCI calculation, but rather diagonalize $\widehat{H}=E_{e l}+\widehat{H}_{S O C}+\widehat{H}_{S S C}$ prior to applying the effective Hamiltonian theory. This point is also valid for the $S=1$ and $S=3 / 2$ systems, although $\widehat{H}_{S S C}$ can be more safely neglected in these cases, especially in complexes for which large ZFSs are observed. For mononuclear complexes, we compare computed values to experimental ones when the SSC contribution to the ZFS can be neglected. When this contribution is expected to play a more important contribution, we do not compare to experiment, but rather focus on the SOC contribution and on the validity of the model Hamiltonians.

\section{II.1.a. $S=1$ and $S=3 / 2$ systems}

For $S=1$ and $S=3 / 2$ systems, the model Hamiltonian which describes the ZFS of orbitally non-degenerate states is simply [Kahn, 1993]:

$$
\widehat{H}_{\bmod 1}=\hat{S} \overline{\bar{D}} \hat{S}
$$

where $\hat{S}$ is the spin operator row or column vector and $\overline{\bar{D}}$ the second-rank ZFS tensor. $\overline{\bar{D}}$ is symmetric and only composed of real numbers. Expanding this Hamiltonian and applying it to the $\left|S, M_{S}\right\rangle$ spin component basis allows one to derive the analytical interaction matrix to be compared to the effective Hamiltonian matrix. Although a similar reasoning can be used for $S=3 / 2$ systems, we will only discuss in details the $S=1$ case. Note that the analytical interaction matrix for $S=3 / 2$ systems is available elsewhere [Maurice et al., 2009]. The analytical interaction matrix is given in Table 1 for $S=1$. As mentioned earlier, the analytical interaction matrix is Hermitian (see Table 1), as any other analytical interaction matrix that will be discussed in this chapter. 
Table 1. Analytical interaction matrix corresponding to the ZFS of $S=1$ systems [Maurice et al., 2009]. $X, Y$ and $Z$ correspond to the Cartesian axes of an arbitrary axis frame.
$\widehat{H}_{\text {mod } 1}$
$|1,-1\rangle$
$|1,0\rangle$
$\langle 1,-1|$
$\frac{1}{2}\left(D_{X X}+D_{Y Y}\right)+D_{Z Z}$
$-\frac{\sqrt{2}}{2}\left(D_{X Z}+i D_{Y Z}\right)$
$\frac{1}{2}\left(D_{X X}-D_{Y Y}\right)+i D_{X Y}$
$\langle 1,0|$
$-\frac{\sqrt{2}}{2}\left(D_{X Z}-i D_{Y Z}\right)$
$D_{X X}+D_{Y Y}$
$\frac{\sqrt{2}}{2}\left(D_{X Z}+i D_{Y Z}\right)$
$\langle 1,1|$

$$
\frac{1}{2}\left(D_{X X}-D_{Y Y}\right)+i D_{X Y} \quad \frac{\sqrt{2}}{2}\left(D_{X Z}-i D_{Y Z}\right) \quad \frac{1}{2}\left(D_{X X}+D_{Y Y}\right)+D_{Z Z}
$$

The c-SOCI calculation delivers wave functions expressed in terms of the spin components of a set of SOF states. To describe the ZFS of an $S=1$ system with $\widehat{H}_{\text {mod1 }}$, the c-SOCI eigenvectors have to be projected onto the spin components of a SOF state, typically the ground state. The norm of the projection can be assessed by looking at the diagonal elements of the overlap matrix between the projected eigenvectors $\left(S\right.$ in $\widehat{H}_{B l o c h}$ or in $\widehat{H}_{\text {des Cloizeaux }}$ ). If the (ground) SOF state is well separated in energy from any other SOF state, the norm of the projections is expected to be close to 1 . Let us choose the example of the $\left[\mathrm{Ni}(\mathrm{HIM} 2-\mathrm{Py})_{2} \mathrm{NO}_{3}\right]^{+}$(HIM2-py = 2-(2-pyridyl)-4,4,5,5-tetramethyl-4,5dihydro-1H-imidazolyl-1-hydroxy) complex (see Figure 1) [Maurice et al., 2009]. 


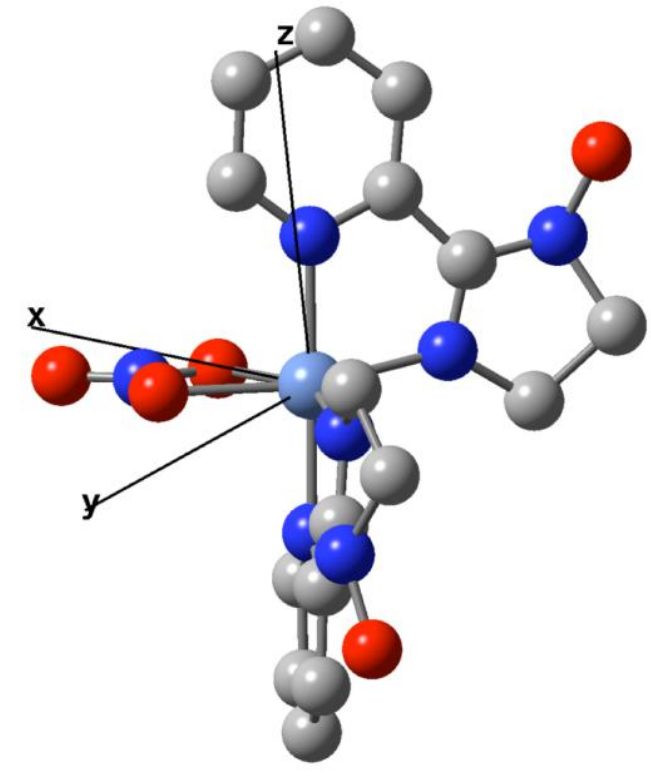

Figure 1. Ball-and-stick representation of a model of the $\left[\mathrm{Ni}(\mathrm{HIM} 2-\mathrm{Py})_{2} \mathrm{NO}_{3}\right]^{+}$complex and its main magnetic axes [Maurice et al., 2009]. The "external" methyl groups have been modelled by hydrogen atoms; all hydrogen atoms are omitted for clarity.

The projected wavefunctions are obtained from the ab initio ones simply by truncation and keeping only the part that concerns the spin components of the $\Psi_{0}$ ground SOF states:

$$
\begin{gathered}
\left|\widetilde{\Psi}_{1}\right\rangle=(0.045+i 0.092)\left|\Psi_{0},-1\right\rangle-(0.668-i 0.724)\left|\Psi_{0}, 0\right\rangle+(0.096+i 0.037)\left|\Psi_{0}, 1\right\rangle \\
\left|\widetilde{\Psi}_{2}\right\rangle=-(0.395-i 0.578)\left|\Psi_{0},-1\right\rangle+(0.062+i 0088)\left|\Psi_{0}, 0\right\rangle-(0.096-i 0.173)\left|\Psi_{0}, 1\right\rangle \\
\left|\widetilde{\Psi}_{3}\right\rangle=(0.701+i 0.026)\left|\Psi_{0},-1\right\rangle-(0.090+i 0.037)\left|\Psi_{0}, 0\right\rangle-(0.519+i 0.472)\left|\Psi_{0}, 1\right\rangle
\end{gathered}
$$

The norms of the projected vectors (prior to orthonormalization) are all larger than 0.99, which perfectly legitimates the use of a spin Hamiltonian in this case. However, prior to validating $\widehat{H}_{m o d 1}$, other tests are necessary: one must show that (i) the effective and analytical interaction matrices match, and (ii) how the extracted tensor component values transform with respect to a change of the axis frame (i.e. that the extracted $\overline{\bar{D}}$ actually transforms as a tensor). The effective interaction matrix that is built with 
$\widehat{H}_{\text {des Cloizeaux }}$ is represented in Table $2\left(E_{1}=0.000, E_{2}=1.529\right.$ and $E_{3}=11.396$, all energies being in $\mathrm{cm}^{-1}$ ).

Table 2. Effective interaction matrix corresponding to the ZFS of the [Ni(HIM2Py) $\left.{ }_{2} \mathrm{NO}_{3}\right]^{+}$complex [Maurice et al., 2009].
$\widehat{H}_{\text {des Cloizeaux }}$
$\left|\Psi_{0},-1\right\rangle$
$\left|\Psi_{0}, 0\right\rangle$
$\left|\Psi_{0}, 1\right\rangle$

$\left\langle\Psi_{0},-1\right|$

6.386

$-0.690+i 0.376$

$-3.734+i 3.134$

$\left\langle\Psi_{0}, 0\right|$

$-0.690-i 0.376$

0.125

$0.690-i .0376$

$\left\langle\Psi_{0}, 1\right|$

$-3.734-i 3.134$

$0.690+i .0376$

6.386

By construction, the effective interaction matrix is Hermitian, has the same eigenvalues as the reference Hamiltonian $\left(\widehat{H}_{r e f}=E_{e l}+\widehat{H}_{S O C}\right)$, and its eigenvectors $\widetilde{\Psi}_{1}, \widetilde{\Psi}_{2}$ and $\widetilde{\Psi}_{3}$ are identical to the projected ab initio eigenvectors up to a given complex phase factor. By the term-by-term comparison of $\widehat{H}_{\text {mod1 }}$ and $\widehat{H}_{\text {des Cloizeaux, }}$ it is immediately clear that both matrices perfectly match, meaning that $\widehat{H}_{\bmod 1}$ is suited to describe the ZFS in this system, as it turns out to be the case for any $S=1$ system with an orbitally nondegenerate ground state. Therefore, the second-rank ZFS tensor can be unambiguously extracted. Diagonalization of this tensor leads to the determination of the magnetic axes $X^{m}, Y^{m}$, and $Z^{m}$, as well as the ZFS parameters:

$$
\begin{gathered}
D=D_{Z^{m} Z^{m}}-\frac{1}{2}\left(D_{X^{m} X^{m}}+D_{Y^{m} Y^{m}}\right) \\
E=\frac{1}{2}\left(D_{X^{m} X^{m}}-D_{Y^{m} Y^{m}}\right)
\end{gathered}
$$

provided that conventions are applied, i.e. $|D|>3 E$ and $E>0$ (or, alternatively $E / D>0$ ). If one uses the transformation matrix $P^{-1}$ that allows expressing $\overline{\bar{D}}$ in the magnetic axis frame (such that $\overline{\bar{D}}^{m}=P^{-1} \overline{\bar{D}} P$ ), one can build again $\widehat{H}_{\text {des }}$ Cloizeaux after computing the cSOCI solutions in this coordinate system, and show that the extracted tensor is diagonal 
and finally that the same ZFS parameters can be extracted. Therefore, we conclude that $\overline{\bar{D}}$ actually transforms as a second-rank tensor and show that the model Hamiltonian $\widehat{H}_{\text {mod } 1}$ is fully valid. In this case, the extracted values for $D$ and $E$ are -10.60 and 0.76 $\mathrm{cm}^{-1}$, respectively [Maurice et al., 2009] and compare well with the most accurate experimental values $\left(-10.15\right.$ and $0.10 \mathrm{~cm}^{-1}$, respectively, from high-field and highfrequency EPR spectroscopy [Rogez et al., 2005]). Other nickel(II) complexes have been studied in a similar way and a good agreement between theory and experiment is generally observed with c-SOCI [Maurice et al., 2009; Maurice et al., 2011b; Costes et al., 2012, Ruamps et al., 2013a; Ruamps et al., 2013b], while density functional theory methods seem to fail for this high-spin $d^{8}$ configuration [Kubica et al., 2013].

A similar reasoning can be applied to $S=3 / 2$ complexes, such as nearly tetrahedral cobalt(II) complexes. The $D$ and $E$ parameters cannot be extracted from the eigenvalues of any reference Hamiltonian, since one only has access to the energy difference between the two Kramer's doublets of interest. On the contrary, the application of the effective Hamiltonian theory unambiguously allows extracting the full ZFS tensor, i.e. determining the magnetic axes and the ZFS parameters. As shown elsewhere [Maurice et al., 2009], $\widehat{H}_{\bmod 1}$ is indeed also perfectly suited to describe the ZFS of $S=3 / 2$ ground states. As an example of application, let us consider the $\left[\mathrm{Co}\left(\mathrm{PPh}_{3}\right) 2 \mathrm{Cl}_{2}\right](\mathrm{Ph}=$ phenyl $)$ complex (see Figure 2) [Maurice et al., 2009]. In this case, the extracted ZFS parameter values are -14.86 and $0.54 \mathrm{~cm}^{-1}$ for $D$ and $E$, respectively [Maurice et al., 2009], which also compares well to the experimental values of -14.76 and $1.14 \mathrm{~cm}^{-1}$, respectively [Krzystek et al., 2004]. Also, note that the energy difference from the two Kramer's doublet of interest relates to the $D$ and $E$ parameters as follows:

$$
\Delta E=2 \sqrt{D^{2}+3 E^{2}}
$$




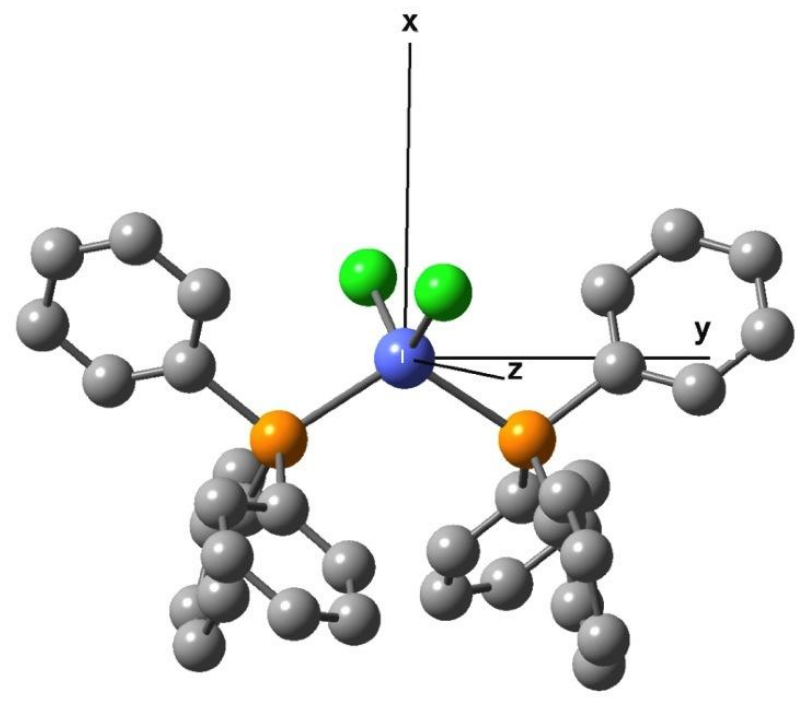

Figure 2. Ball-and-stick representation of the $\left[\mathrm{Co}\left(\mathrm{PPh}_{3}\right) 2 \mathrm{Cl}_{2}\right]$ complex and its main magnetic axes [Maurice et al., 2009]. All the hydrogen atoms are omitted for clarity.

In both nickel(II) and cobalt(II) examples described above, the computational methodology was based on SA-CASSCF calculations with quite large active spaces, small sets of SOF states (4T and 7Q, respectively), and CASPT2 correlated energies [Maurice et al., 2009]. As a recommendation, one may note that enlarging the set of SOF states does not systematically improve the results: on the one hand more SOC excitations are accounted for, on the other hand averaging artefacts are introduced, which may result for instance in a poor orbital set for describing the ground state. Therefore, one should make the compromise of describing as best as possible the stronger SOC interactions, i.e. balance between the number of possible excitations and the accurate description of the involved states. As a conclusion concerning the ab initio methodology, one may say that c-SOCI methods do not constitute a "black-box machinery" to compute ZFSs.

\section{II.2.b. $S=2$ and $S=5 / 2$ systems}

For $S=2$ and $S=5 / 2$ complexes, additional operators must be introduced in the model Hamiltonian to achieve a complete description of the ZFS: 


$$
\widehat{H}_{\text {mod } 2}=\hat{S} \overline{\bar{D}} \hat{S}+\sum_{q=-4}^{4} B_{4}^{q} \hat{O}_{4}^{q}
$$

where $q$ may be odd, and where the $\hat{O}_{4}^{q}$ operators are extended Stevens operators [Stevens, 1952; Altshuler and Kozyrev, 1974; Abragam and Bleaney, 1986; Rudowicz and Chung, 2004]. This Hamiltonian is valid in any arbitrary axis frame for $S=2$ and $S=5 / 2$ complexes. When the ground SOF state is well separated in energy from the excited states, the fourth-rank spin $\hat{O}_{4}^{q}$ operators have a very small effect on the effective interactions of the model $\widehat{H}_{\text {mod } 1}$. Therefore, one can first find the main anisotropy axes by extracting $\overline{\bar{D}}$ from the comparison of the effective interaction matrix and the analytical one that is obtained with $\widehat{H}_{\bmod 1}$, and then compute the effective interaction matrix in this frame after a second c-SOCI calculation. In this case, the model Hamiltonian reduces to:

$$
\widehat{H}_{\text {mod3 }}=\sum_{n=0}^{4} \sum_{k=2}^{4} B_{k}^{n} \widehat{O}_{k}^{n}
$$

where $k$ and $n$ must be even, and the $\hat{O}_{k}^{n}$ operators are standard Stevens operators. Note that $B_{2}^{0}=D / 3$ and that $B_{2}^{2}=E$, i.e. that $B_{2}^{0}$ is a second-rank axial ZFS parameter, while $B_{2}^{2}$ is a rhombic one. As shown elsewhere, this two-step procedure leads to the unambiguous extraction of the main magnetic axes and of the five $B_{k}^{n}$ parameters $\left(B_{2}^{0}\right.$, $B_{2}^{2}, B_{4}^{0}, B_{4}^{2}$, and $B_{4}^{4}$ ) [Maurice et al., 2010a]. Indeed, in the magnetic axis frame, the effective interaction matrix is in almost perfect correspondence with the analytical one derived for $\widehat{H}_{\bmod 3}$ with only some negligible deviations, typically not larger than 0.01 $\mathrm{cm}^{-1}$. These deviations can be considered as numerical noise, and do not significantly alter the extracted $B_{k}^{n}$ values. Note that if one wants to neglect the $B_{4}^{n}$ parameters, i.e. introduce only second-rank spin operators in the model Hamiltonian, as in $\widehat{H}_{\text {mod1 }}$, it is important to check a priori that the $k=4$ terms are not important to insure that the model and effective interaction matrices match. As will be discussed in section III.2, the fourth-rank Stevens parameters relate to the near-degeneracy of spin-components of different SOF states. Therefore, one should not neglect them in such situations, as for instance in nearly-octahedral manganese(III) complexes [Maurice et al., 2010a]. Note that however, the Jahn-Teller effect tends to largely remove the near-degeneracy 
between the two lowest orbital configurations in this case, which explains for instance why the $B_{4}^{n}$ parameters are not crucial to describe the ZFS in the $\left.[\gamma \text {-Mn(acac })_{3}\right]$ (acac=acetylacetonato) complex (see Figure 3) [Maurice, 2011], for which the first coordination sphere is tetragonally elongated.

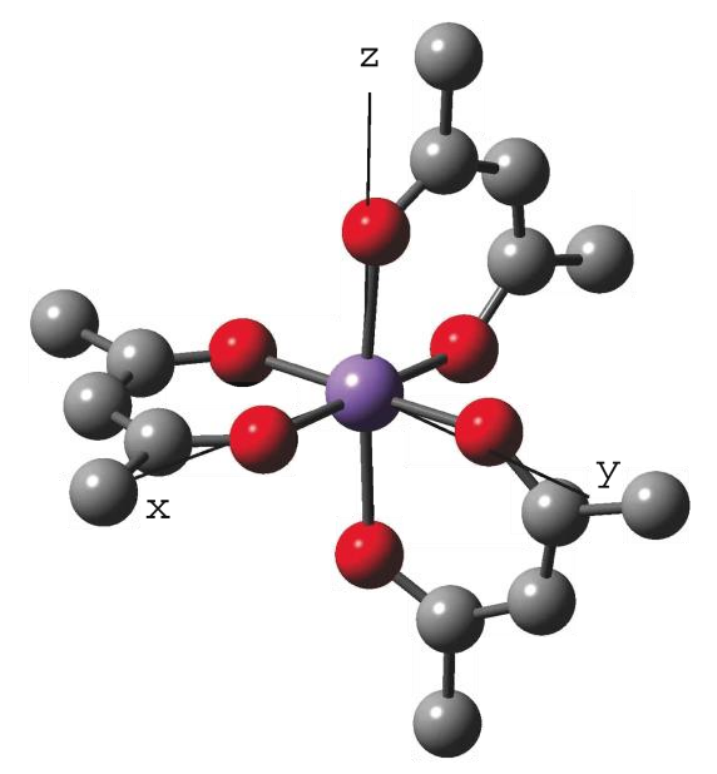

Figure 3. Ball-and-stick representation of the $\left[\gamma-\mathrm{Mn}(\mathrm{acac})_{3}\right]$ complex and its main magnetic axes [Maurice, 2011]. All the hydrogen atoms are omitted for clarity.

In many cases of experimental interest, $\widehat{H}_{\bmod 1}$ is perfectly suited to describe the ZFS of $S=2$ and $S=5 / 2$ complexes.

\section{II.2. Binuclear Complexes}

Prior to introducing the model Hamiltonians that can be used to describe the ZFS in binuclear complexes, it is worth introducing the HDV Hamiltonian [Heisenberg, 1928; Dirac, 1929; Van Vleck, 1945], which may be expressed in a "multispin" picture, i.e. by considering local spin operators that are to be applied within the basis of local spin components, i.e. within the uncoupled spin basis:

$$
\widehat{H}_{H D V}^{\text {uncoupled }}=J \hat{S}_{a} \cdot \hat{S}_{b}
$$

where $J$ is the isotropic coupling constant, and $\hat{S}_{a}$ and $\hat{S}_{b}$ are spin operator column vectors. Note that various expressions coexist in the literature, depending of a factor 
that is applied to this Hamiltonian (here 1 , but one may find -1 or more often -2 ). It can easily be shown that $\widehat{H}_{\bmod 1}$ can be also written in terms of spin operators that lead to a diagonal analytical interaction matrix if one works within the basis of spin eigenfunctions, i.e. within the coupled spin basis:

$$
\widehat{H}_{H D V}^{\text {coupled }}=\frac{J}{2} \sum_{S}\left(\hat{S}^{2}-\hat{S}_{a}^{2}-\hat{S}_{b}^{2}\right)
$$

where $S$ ranges between $\left|S_{a}-S_{b}\right|$ and $S_{a}+S_{b}, \hat{S}$ is the spin operator associated to each coupled spin state, and $S_{a}$ and $S_{b}$ are the local spins on the $a$ and $b$ sites. Therefore, it is clear that $\widehat{H}_{H D V}$ splits the coupled spin states, which may further be the subject of ZFS and mixings when anisotropic effective interactions are considered in the model Hamiltonian. Such ZFSs and "spin mixings" can be effectively described in two different ways that work in the coupled and uncoupled basis, respectively, and which are classified as "giant-spin" or "block-spin" models, and "multispin" ones.

\section{II.2.a. Giant-spin and block-spin Hamiltonians}

If the isotropic coupling constant plays a much more important role on the effective interaction matrix than the spin mixings, which is usually referred to as the "strongexchange limit" [Boča, 1999], very simple models can be used to describe the lowenergy spectrum. If only one spin "block" has to be described and that its magnetic axis frame is considered, one can use a very simple giant-spin Hamiltonian that is similar to $\widehat{H}_{\bmod 3}$ :

$$
\widehat{H}_{\text {giant spin }}^{\text {coupled }}=\sum_{n=0}^{k} \sum_{k=2}^{x} B_{k}^{n} \hat{O}_{k}^{n}
$$

where $x=2 S$ if $S$ is even or $x=2 S-1$ if $S$ is odd, and $k$ and $n$ are even.

The simplest case is the ZFS of an $S=1$ spin state resulting from the coupling of two local spins $S_{a}=S_{b}=1 / 2$. One typical example of such a situation is copper acetate monohydrate (see Figure 4) [Maurice et al., 2011a]. 


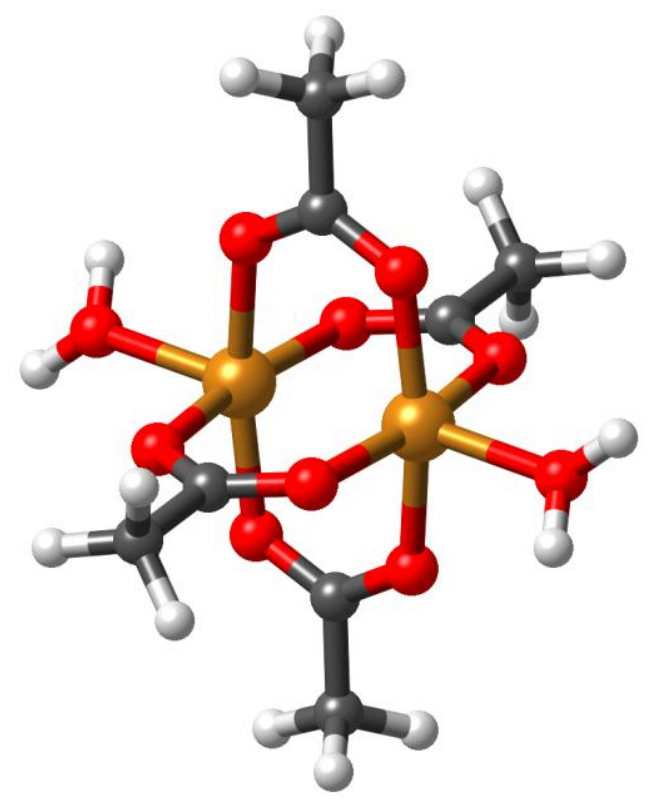

Figure 4. Ball-and-stick representation of copper acetate monohydrate [Maurice et al., 2011a].

In this system, it is crucial to account for both SOC and SSC to compute the ZFS of the excited ${ }^{3} \mathrm{~A}_{1 \mathrm{u}}$ SOF state. As shown by Maurice et al [Maurice et al., 2011a], the treatment of SOC requires a special attention to the correlated energies appearing on the diagonal elements of the $\widehat{H}=E_{e l}+\widehat{H}_{S O C}+\widehat{H}_{S S C}$ matrix (see Table 3 ). The reference wave functions were obtained with SA-CASSCF(18/10) calculations (see [Maurice et al., 2011a] for more details). As can be seen in Table 3, second-order perturbation theory does not describe sufficiently well the SOF excitation energies, as well as variational approaches with limited configuration interaction spaces (the DDCI1 calculations include in this case only the $1 h$ and $1 p$ excitations, while the DDCI2 ones also account for the $1 h 1 p, 2 h$, and $2 p$ excitations). Although the DCCI2 level appears to lead to an almost converged value of $D$ when only $\widehat{H}_{S O C}$ is considered, it is essentially due to error cancellations (more details are given on this in [Maurice et al., 2011a]). The best value, obtained with the DDCI3 energies, is in exceptional agreement with experiment $\left(D_{\text {expt. }}=-0.335 \mathrm{~cm}^{-1}\right.$ [Ozarowski, 2008]). The rhombic $E$ parameter value is very small, $0.006 \mathrm{~cm}^{-1}$ with the DDCI3 energies (in good agreement with $E_{\text {expt. }}=0.01 \mathrm{~cm}^{-1}$ ), and will not be discussed in details here. 
Table 3. Computed axial ZFS $D$ parameters as a function of the correlated energies used on the diagonal of the contracted configuration interaction matrices, and of the operators introduced in the Hamiltonian [Maurice et al., 2011a].

\begin{tabular}{cccc}
\hline$E_{e l}$ & $\widehat{H}_{S O C}$ & $\widehat{H}_{S S C}$ & $\widehat{H}_{S O C}+\widehat{H}_{S S C}$ \\
\hline SA-CASSCF & -0.017 & -0.118 & -0.137 \\
NEVPT2 & -0.026 & -0.118 & -0.144 \\
DDCI1 & 0.005 & -0.118 & -0.115 \\
DDCI2 & -0.172 & -0.118 & -0.291 \\
DDCI3 & -0.200 & -0.118 & -0.319 \\
\hline
\end{tabular}

One should stress that in this particular case, i.e. the $d^{9}-d^{9}$ configuration, computing the ZFS of the triplet block happened to be particularly challenging, but it may not be the case for other configurations. Another system that has been studied within the giantspin approach is the $\left[\mathrm{Ni}_{2}(\mathrm{en})_{4} \mathrm{Cl}_{2}\right]^{2+}$ (en=ethylenediamine) complex (see Figure 5) [Maurice et al., 2010b; Maurice et al., 2010c]. Although this system does not fall within the strong-exchange limit, it is possible to build two effective Hamiltonians in the basis of the spin components of the $S=2, S=1$ and $S=0$ spin components, one in which spin mixings are set to zero, and one in which these spin mixings are allowed [Maurice et al., 2010c; Maurice, 2011]. From this theoretical study, it was shown that, in the magnetic axis frame, $\widehat{H}_{\text {giant spin }}^{\text {coupled }}$ can describe the ZFS of both the $S=2$ and $S=1$ blocks in the absence of spin mixing, and that the spin mixings can be described using additional operators [Maurice et al., 2010c] (in this case, due to a symmetry centre, the spin mixings concern the $S=0$ and some $S=2$ spin components). In other words, one can define a block spin Hamiltonian which describes the isotropic coupling and the ZFSs of the different blocks in the absence of spin mixing, and the spin-mixing effects can actually be introduced inside the different spin blocks, i.e. one can use a block-diagonal analytical interaction matrix to describe the entire low-energy spectrum [Maurice, 2011]. The comparison with experimental data is complicated since all the studies reported so far neglected some, and usually different, effective interactions in the model Hamiltonians [Ginsberg et al., 1972; Journaux et al., 1978; Joung et al., 1979; Herchel et al., 2007]. One can just mention that the best computed value for $D_{2}$, i.e. the axial ZFS parameter of the $S=2$ block, is in good semi-quantitative agreement with the experimental one [Herchel et al., 2007] ( -3.0 vs. $-1.8 \mathrm{~cm}^{-1}$, respectively). 


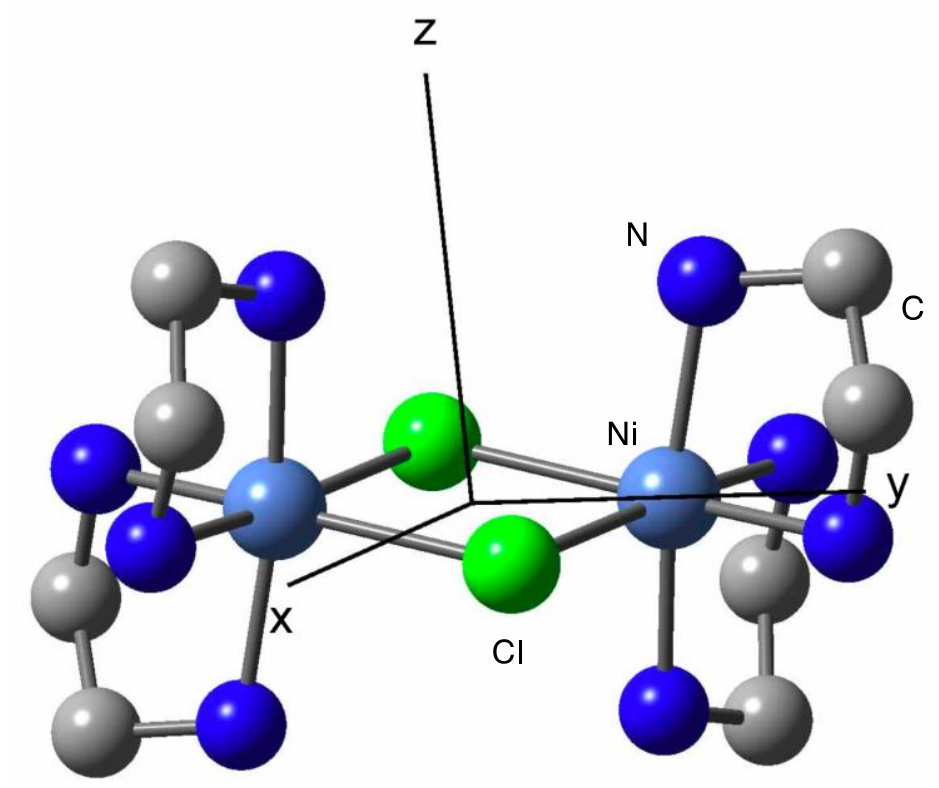

Figure 5. Ball-and-stick representation of the $\left[\mathrm{Ni}_{2}(\mathrm{en})_{4} \mathrm{Cl}_{2}\right]^{2+}$ complex and its $S=2$ block main magnetic axes. All the hydrogen atoms are omitted for clarity.

Another study of the $d^{8}-d^{8}$ configuration, related to the strong-exchange limit, concerned model complexes [Ruamps et al., 2014]. It was shown that, contrary to what is often proposed, no simple relations appear between the ZFS parameters of the $S=2$ and $S=1$ blocks, which can also be analysed within a multispin picture (vide infra). Also, it is clear that giant-spin and block-spin Hamiltonians may not be relevant in the weak-exchange limit, i.e. when $J$ becomes negligible, since spin mixings cannot be considered as a perturbation in such a case. In principle, one should in this case consider a multispin model, the extraction of which is far from being straightforward, as will be shown later.

\section{II.2.b. Multispin Hamiltonians}

As in section II.1, we will introduce progressively the complexity of the multispin Hamiltonian in binuclear complexes. Let us start with the easiest case of two coupled $S=1 / 2$ centres, as in the $d^{9}-d^{9}$ configurations for instance. The model Hamiltonian which 
is commonly used includes an isotropic coupling terms $\left(\widehat{H}_{H D V}\right)$ plus an anisotropy tensor [Khan, 1993]:

$$
\widehat{H}_{\text {multispin }\left(S_{a}=S_{b}=1 / 2\right)}^{\text {uncoupled }}=J \hat{S}_{a} \cdot \hat{S}_{b}+\hat{S}_{a} \overline{\bar{T}}_{a b} \hat{S}_{b}=J \hat{S}_{a} \cdot \hat{S}_{b}+\hat{S}_{a} \overline{\bar{D}}_{a b} \hat{S}_{b}+\bar{d}_{a b} \hat{S}_{a} \times \hat{S}_{b}
$$

where $\overline{\bar{T}}_{a b}$ is a second-rank tensor that is neither symmetric nor antisymmetric in the general case, $\overline{\bar{D}}_{a b}$ is the symmetric anisotropy exchange tensor, and $\bar{d}_{a b}$ is the Dzyaloshinskii-Moriya term [Dzyaloshinskii, 1958; Moriya, 1960], that can also be referred to as the antisymmetric exchange pseudo-vector. As mentioned earlier, computing $\overline{\bar{D}}_{a b}$ may turn into a real nightmare [Maurice et al., 2011a], but the semiquantitative determination of $\bar{d}_{a b}$ is much less demanding, since it can be obtained from CASSCF(2/2) + c-SOCI calculations [Maurice et al., 2010d; Pradipto et al., 2012]. The analytical interaction matrix built in the uncoupled basis is represented in Table 4 .

Table 4. Analytical interaction matrix corresponding to $\widehat{H}_{\text {multispin }}^{\text {uncoupled }}$ when $S_{a}=S_{b}=1 / 2$ [Maurice et al., 2010d]. A shortened notation $\left|M_{S_{a^{\prime}}}, M_{S_{b}}\right\rangle$ is used for the $\left|S_{b}, M_{S_{a}}, S_{b}, M_{S_{b}}\right\rangle$ uncoupled functions. $X, Y$ and $Z$ correspond to the Cartesian axes of an arbitrary axis frame.

\begin{tabular}{|c|c|c|c|c|}
\hline$\widehat{H}_{\text {multispin }}^{\text {uncoupled }}$ & $|-1 / 2,-1 / 2\rangle$ & $|-1 / 2,1 / 2\rangle$ & $|1 / 2,-1 / 2\rangle$ & $|1 / 2,1 / 2\rangle$ \\
\hline$\langle-1 / 2,-1 / 2|$ & $\frac{1}{4}\left(J+T_{Z Z}\right)$ & $-\frac{1}{4}\left(T_{Z X}+i T_{Z Y}\right)$ & $-\frac{1}{4}\left(T_{X Z}+i T_{Y Z}\right)$ & $\begin{array}{l}\frac{1}{4}\left[T_{X X}-T_{Y Y}\right. \\
\left.+i\left(T_{X Y}+T_{Y X}\right)\right]\end{array}$ \\
\hline$\langle-1 / 2,1 / 2|$ & $-\frac{1}{4}\left(T_{Z X}-i T_{Z Y}\right)$ & $-\frac{1}{4}\left(J+T_{Z Z}\right)$ & $\begin{array}{l}\frac{1}{4}\left[2 J+T_{X X}+T_{Y Y}\right. \\
\left.+i\left(T_{Y X}-T_{X Y}\right)\right]\end{array}$ & $\frac{1}{4}\left(T_{X Z}+i T_{Y Z}\right)$ \\
\hline$\langle 1 / 2,-1 / 2|$ & $-\frac{1}{4}\left(T_{X Z}-i T_{Y Z}\right)$ & $\begin{array}{l}\frac{1}{4}\left[2 J+T_{X X}+T_{Y Y}\right. \\
\left.-i\left(T_{Y X}-T_{X Y}\right)\right]\end{array}$ & $-\frac{1}{4}\left(J+T_{Z Z}\right)$ & $\frac{1}{4}\left(T_{Z X}+i T_{Z Y}\right)$ \\
\hline$\langle 1 / 2,1 / 2|$ & $\begin{array}{l}\frac{1}{4}\left[T_{X X}-T_{Y Y}\right. \\
\left.-i\left(T_{X Y}+T_{Y X}\right)\right]\end{array}$ & $\frac{1}{4}\left(T_{X Z}-i T_{Y Z}\right)$ & $\frac{1}{4}\left(T_{Z X}-i T_{Z Y}\right)$ & $\frac{1}{4}\left(J+T_{z Z}\right)$ \\
\hline
\end{tabular}

One can also express this analytical interaction matrix with the $\overline{\bar{D}}_{a b}$ tensor and the $\bar{d}_{a b}$ pseudo vector by using the $D_{i i}=T_{i i}, D_{i j}=1 / 2\left(T_{i j}+T_{\mathrm{ji}}\right), d_{\mathrm{X}}=1 / 2\left(T_{Y Z}-T_{Z Y}\right), d_{\mathrm{Y}}=1 / 2\left(T_{Z X}-T_{X Z}\right)$, 
and $d_{Z}=1 / 2\left(T_{X Y}-T_{Y X}\right)$ relations [Maurice et al., 2010d]. The analytical interaction matrix can be transformed into the coupled basis as follows:

$$
\widehat{H}_{\text {multispin }}^{\text {coupled }}=U^{T} \widehat{H}_{\text {multispin }}^{\text {uncoupled }} U
$$

where $U^{T}$ is the transpose of the change of basis matrix $U$. The matrix elements of $U$ are given by the appropriate Clebsch-Gordan coefficients [Boča, 1999]. When $\widehat{H}_{\text {multispin }}$ is expressed in the coupled basis, the $\overline{\bar{D}}_{a b}$ tensor relates to the splitting and mixing of the $S=1$ block (i.e. its ZFS in the strong-exchange limit), while the $\bar{d}_{a b}$ pseudo-vector introduces spin-mixings between the $S=1$ spin components and the $S=0$ one. This type of S/S+1 spin mixing is not always symmetry allowed (the symmetry rules are available elsewhere [Buckingham et al., 1982]). One may just recall that if the system contains a symmetry centre, as it is the case for copper acetate monohydrate, the $\bar{d}_{a b}$ pseudovector is null [Moriya, 1960]. Therefore, in this system, as in any other $d^{9}-d^{9}$ binuclear system, a simple relation appears between $\overline{\bar{D}}_{1}$ and $\overline{\bar{D}}_{a b}$ [Maurice et al., 2011a]:

$$
\overline{\bar{D}}_{1}=\frac{1}{2} \overline{\bar{D}}_{a b}
$$

By studying model copper(II)-copper(II) complexes, Maurice et al. showed that $\widehat{H}_{\text {multispin }}$ is perfectly valid to describe the isotropic coupling and the ZFSs when $S_{a}=S_{b}=1 / 2$ [Maurice et al., 2010d]. Owing to the effective Hamiltonian theory, all the model parameter values can be theoretically extracted, while it appears complicated to properly distinguish between the symmetric and antisymmetric exchange terms from the outcomes of experiments. Moreover, since one can obtain good semi-quantitative estimates of the $\bar{d}_{a b}$ pseudo-vector components from CASSCF(2/2) + c-SOCI calculations [Maurice et al., 2010d; Pradipto et al., 2012], antisymmetric exchange is essentially due to the direct SOC between the $S=1$ and $S=0$ spin components.

When $S_{a}=1$ and $S_{b}=1 / 2$, another term must be added to the phenomenological model Hamiltonian, related to the single-ion anisotropy of site $a$ [Khan, 1993]:

$$
\widehat{H}_{\text {multispin }\left(S_{a}=1, S_{b}=1 / 2\right)}^{\text {uncoupled }}=J \hat{S}_{a} \cdot \hat{S}_{b}+\hat{S}_{a} \overline{\bar{D}}_{a} \hat{S}_{a}+\hat{S}_{a} \overline{\bar{D}}_{a b} \hat{S}_{b}+\bar{d}_{a b} \hat{S}_{a} \times \hat{S}_{b}
$$


In this case, both the $\overline{\bar{D}}_{a}$ and $\overline{\bar{D}}_{a b}$ symmetric tensors affect the $S=3 / 2$ block, while $\bar{d}_{a b}$ relates to the $\mathrm{S} / \mathrm{S}+1$ spin mixings, as usual.

The situation is drastically complicated when considering the $S_{a}=S_{b}=1$ case, e.g. nickel(II)-nickel(II) complexes. The following model Hamiltonian was used for decades to interpret experimental data [Ginsberg et al., 1972; Journaux et al., 1978; Joung et al., 1979; Herchel et al., 2007]:

$$
\widehat{H}_{\text {multispin }\left(S_{a}=S_{b}=1\right)}^{\text {uncoupled }}=J \hat{S}_{a} \cdot \hat{S}_{b}+\hat{S}_{a} \overline{\bar{D}}_{a} \hat{S}_{a}+\hat{S}_{b} \overline{\bar{D}}_{b} \hat{S}_{b}+\hat{S}_{a} \overline{\bar{D}}_{a b} \hat{S}_{b}+\bar{d}_{a b} \hat{S}_{a} \times \hat{S}_{b}
$$

The validity of this model Hamiltonian was assessed by c-SOCI calculations and the effective Hamiltonian theory in 2010 by Maurice et al. [Maurice et al., 2010b]. It was shown that many terms of the effective interaction matrix which were obtained for the $\left[\mathrm{Ni}_{2}(\mathrm{en})_{4} \mathrm{Cl}_{2}\right]^{2+}$ complex were not associated to any model parameter in the analytical interaction matrix. In order to reproduce all features of the effective Hamiltonian matrix one must actually introduce a symmetric fourth-rank exchange tensor, $\boldsymbol{D}_{a a b b}$, in the model Hamiltonian, leading to:

$$
\widehat{H}_{\text {multispin }\left(S_{a}=S_{b}=1\right)}^{\text {uncoupled }}=J \hat{S}_{a} \cdot \hat{S}_{b}+\hat{S}_{a} \overline{\bar{D}}_{a} \hat{S}_{a}+\hat{S}_{b} \overline{\bar{D}}_{b} \hat{S}_{b}+\hat{S}_{a} \overline{\bar{D}}_{a b} \hat{S}_{b}+\hat{S}_{a} \otimes \hat{S}_{a} \boldsymbol{D}_{a a b b} \hat{S}_{b} \otimes \hat{S}_{b}
$$

if $\bar{d}_{a b}$ is null. The extraction of the $\boldsymbol{D}_{a a b b}$ components is not straightforward even with all information contained in the 9x9 effective interaction matrix; one should thus consider relations between these components, as done in a study of model complexes [Ruamps et al., 2014]. One should thus stress that the spin mixings between the $S=2$ and $S=0$ spin components, mentioned in section II.2.a can be interpreted in terms of the parameters of the multispin Hamiltonian after transforming the analytical interaction matrix to the coupled basis: these terms actually relates to all symmetric tensors of $\widehat{H}_{\text {multispin }}$, i.e. $\overline{\bar{D}}_{a}, \overline{\bar{D}}_{b}, \overline{\bar{D}}_{a b}$, and $\boldsymbol{D}_{a a b b}$ [Maurice et al., 2010c].

Another interesting point which is worth mentioning here is that one may be interested in computing only the local anisotropy tensors, i.e. $\overline{\bar{D}}_{a}$ and $\overline{\bar{D}}_{b}$. Various strategies exist, (i) one may replace one of the two magnetic centres by a model potential [Maurice et al., 2010b]or by a diamagnetic ion [Maurice et al., 2010b; Bogdanov et al., 2013, Maurice et al., 2013a], (ii) one may also consider its lowest-energy closed-shell configuration [Ruamps 
et al., 2014], (iii) and another method considers local excitations while keeping the other site in its lowest-energy open-shell configuration [Retegan et al., 2014]. This last strategy is meant to be the most accurate approach. From the study of model complexes, it was shown that the local anisotropy parameters that can be obtained in these various ways are in very close agreement with those obtained from the extraction of the interactions of $\widehat{H}_{\text {multispin }}$ [Ruamps et al., 2014]. In conclusion, if one wants to estimate local anisotropy parameters or local magnetic axes, these approaches can be safely considered. It is also worth mentioning here is that in the general case, the $S / S+1$ spin mixings do not arise solely from $\bar{d}_{a b}$ : the mismatch between the local magnetic axes of the local $\overline{\bar{D}}_{a}$ and $\overline{\bar{D}}_{b}$ tensors also affects the effective couplings related to these mixings. This can be easily shown by considering $D_{a}=D_{b} \neq 0, E_{a}=E_{b}=0$, and an angle $2 \alpha$ between coplanar local $Z_{a, b}^{m}$ axes. If one builds the correspond model interaction matrix within the uncoupled basis, and transforms it to the coupled one, the $S / S+1$ spin mixing terms are found proportional to $D_{a} \sin 2 \alpha$ (the details of the derivation are not given here for a sake of simplicity). It is thus clear that these terms vanish for $\alpha=0$, as it is the case for centrosymmetric complexes. Therefore, the local anisotropy tensors can be affected in the general case by the $S / S+2$ and to $S / S+1$ spin mixings. Furthermore, note that one should also never neglect these terms within the weak-exchange limit, contrary to what was done for instance to interpret the low-energy spectrum of a cobalt(II)-cobalt(II) complex [Ostrovsky et al., 2002]. From the perspective of modelling, it is not clear yet whether the model Hamiltonian used for the $S_{a}=S_{b}=1$ case is directly applicable to any other configuration. Actually higher-rank tensors could be necessary to reproduce all ZFS features for higher local spins [Maurice et al., 2013b], such as for instance a sixthrank tensor in the $S_{a}=S_{b}=3 / 2$ case.

\section{Magneto-structural correlations}

Magneto-structural correlations are particularly useful for chemists as they give clues to tune the properties of a system and pave the way for the rational design of new magnetic systems with predetermined properties. In the field of ZFS, they can be established from correlations of molecular geometry features with the experimental values of the parameters, as was done by Titiš and Boča in nickel(II) and cobalt(II) mononuclear complexes [Titiš and Boča, 2010; Titiš and Boča, 2011]. Here, we will only 
discuss magneto-structural correlations deduced from the analysis of $a b$ initio results, or from combined $a b$ initio/crystal-field studies.

\section{III.1. Magneto-structural correlations based on $a b$ initio calculations}

Due to the lack of intuition on the role of distortions on the $\bar{d}_{a b}$ pseudo-vector components, Maurice et al. studied the effects of two angular distortions on the DM vector components on model $\left[\mathrm{Cu}_{2} \mathrm{O}\left(\mathrm{H}_{2} \mathrm{O}\right)_{6}\right]^{2+}$ complexes (see Figure 6) [Maurice et al., 2010d]. This study was based on $\operatorname{CASSCF}(2 / 2)+$ c-SOCI calculations, and made use of the effective Hamiltonian theory, as mentioned in section II.2.b.
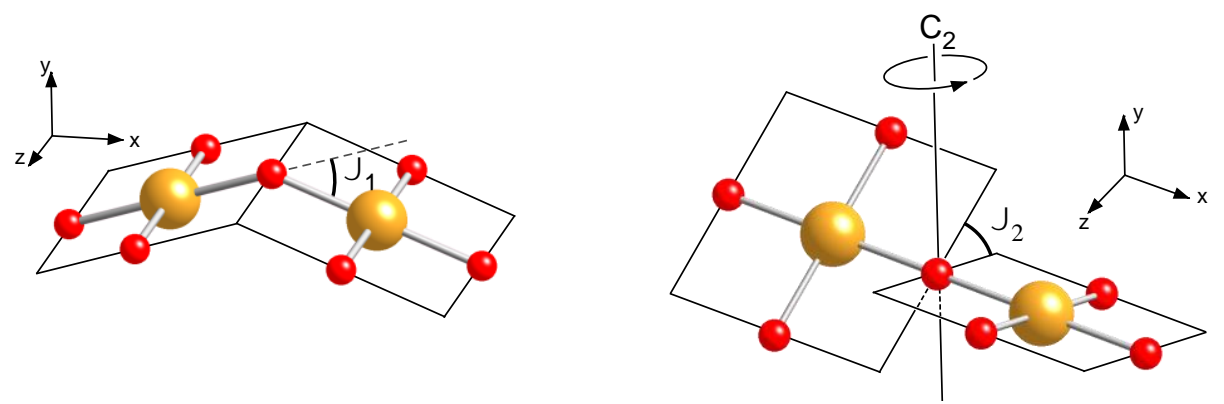

Figure 6. Ball-and-stick representation of model $\left[\mathrm{Cu}_{2} \mathrm{O}\left(\mathrm{H}_{2} \mathrm{O}\right)_{6}\right]^{2+}$ complexes and the deformation angles that were applied to them [Maurice et al., 2010d]. All the hydrogen atoms are omitted for clarity.

The norm of the $\bar{d}_{a b}$ pseudo-vector as a function of the $\vartheta_{1}$ and $\vartheta_{2}$ deformation angles is represented in Figure 7. 


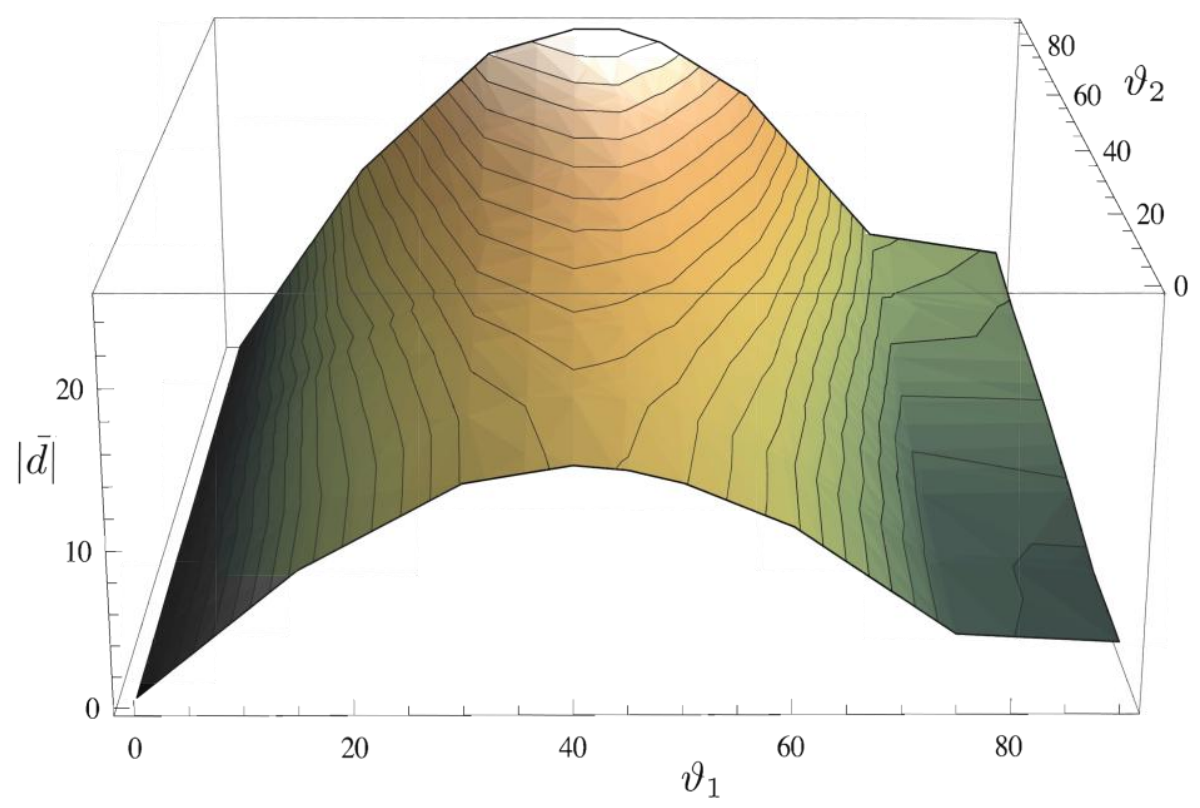

Figure 7. Norm of the $\bar{d}_{a b}$ pseudo-vector as a function of the $\vartheta_{1}$ and $\vartheta_{2}$ deformation angles (see Figure 6 for the definition of these angles) [Maurice et al., 2010d].

In this study, the only point for which the $\bar{d}_{a b}$ pseudo-vector is null by symmetry corresponds to the $\vartheta_{1}=\vartheta_{2}=0$ case, for which the system possesses a symmetry centre. Another interesting point refers to $\vartheta_{1}=\pi / 2$ and $\vartheta_{2}=0$. In this case, there is no atomic orbital contribution from the copper(II) centres, and as a consequence, the only important contribution to this norm comes from the bridging oxygen centre. As the $\bar{d}_{a b}$ pseudo-vector is far from being null in this case $\left(\left|\bar{d}_{a b}\right|=3.6 \mathrm{~cm}^{-1}\right.$ [Maurice et al., 2010d]), the (essentially) closed-shell bridging oxygen contributes to the norm of the $\bar{d}_{a b}$ pseudo-vector, as highlighted by Moskvin in 2007 [Moskvin, 2007]. Other studies concerning single-ion anisotropies exist in the literature, among which one may quote the extensive one of Gomez-Coca et al. [Gomez-Coca et al., 2013], but these will not be discussed here. Instead, we will explore the cases of mononuclear complexes for which $a b$ initio calculations are used to extract crystal-field parameters.

\section{III.2. Magneto-structural correlations based on crystal-field models and $a b$ initio calculations}

Joint $a b$ initio and crystal-field studies are important to validate the equations derived from the crystal-field theory and to interpret the outcomes of experiments in a simple way. It is commonly practiced on mononuclear complexes, although equations can also 
be derived in binuclear complexes, as was done for instance in the case of copper acetate monohydrate [Maurice et al., 2011a. In this section, we shall illustrate the procedure for the $d^{8}$ and $d^{4}$ configurations, using ab initio calculations on nearly-octahedral $\left[\mathrm{Ni}(\mathrm{NCH})_{6}\right]^{2+}$ and $\left[\mathrm{Mn}(\mathrm{NCH})_{6}\right]^{3+}$ model complexes. In both cases, we define the axial deformation parameter as:

$$
\tau_{a x}=\frac{2 d\left(T M, N_{Z}\right)}{d\left(T M, N_{X}\right)+d\left(T M, N_{Y}\right)}
$$

and the rhombic deformation as:

$$
\tau_{r h}=\frac{d\left(T M, N_{Y}\right)}{d\left(T M, N_{X}\right)}
$$

The mean $d(\mathrm{TM}, \mathrm{N})$ distance is $2.054 \AA$ and $2.061 \AA$ for TM $=\mathrm{Ni}$ [Maurice, 2011] and TM $=\mathrm{Mn}$ [Maurice et al., 2010a], respectively, while all $d(\mathrm{TM}, \mathrm{C})$ parameters are fixed to $1.155 \AA$ and all the $d(\mathrm{C}, \mathrm{H})$ ones to $1.083 \AA$. Minimal CASSCF calculations have been carried out with the five d-orbitals and $8(\mathrm{Ni})$ or $5(\mathrm{Mn})$ electrons within the active space. Note that in the formulae that are presented here, monoelectronic $\zeta$ SOC constants are considered. These constants are always positive and can be converted into polyelectronic $\lambda$ ones by using the following relation:

$$
\lambda= \pm \frac{\zeta}{2 S}
$$

where $S$ is the total spin of the ground SOF free-ion multiplet.

It is easy to show, as done in the textbook of Abragam and Bleaney [Abragam and Bleaney, 1986], that for axially distorted systems:

$$
D=-\frac{\zeta^{2}}{\Delta_{1}}+\frac{\zeta^{2}}{\Delta_{2}}
$$

where $\Delta_{1}$ is the ${ }^{3} \mathrm{~B}_{1 \mathrm{~g}} \rightarrow{ }^{3} \mathrm{~B}_{2 \mathrm{~g}}$ excitation energy and $\Delta_{2}$ corresponds to the ${ }^{3} \mathrm{~B}_{1 \mathrm{~g}} \rightarrow{ }^{3} \mathrm{E}_{\mathrm{g}}$ excitation energy. The derivation of this equation is based on a model space containing the 
spin components of the ground $\mathrm{SOF}^{3} \mathrm{~B}_{1 \mathrm{~g}}$ state, while the external space consists of the spin components of the lowest two excited SOF states, namely ${ }^{3} \mathrm{~B}_{2 \mathrm{~g}}$ and ${ }^{3} \mathrm{E}_{\mathrm{g}}$, which are essentially singly-excited states with respect to ${ }^{3} \mathrm{~B}_{1 \mathrm{~g}}$. To check the correlation between the $a b$ initio results and the outcomes of the crystal-field model, we substitute the $a b$ initio $\Delta_{1}$ and $\Delta_{2}$ values in the expression for $D$, and take the SOC constant of the free $\mathrm{Ni}^{2+}$ ion, $648 \mathrm{~cm}^{-1}$ (see Table 5). As can be seen, a good correlation appears between the ab initio and the crystal-field $D$ values $\left(D_{\mathrm{CF}}\right)$ : the trend line that passes through the origin, as forced by symmetry, leads to $D_{\mathrm{CF}}=1.320 \mathrm{D}$ with $R^{2}=0.9969$. This shows that accounting for covalency effects by applying a reduction factor of 0.87 brings the $D_{\mathrm{CF}}$ values in perfect agreement with the ab initio ones along the whole curve. Therefore, the crystalfield formula presented above is fully supported by c-SOCI calculations that consider the spin components of four SOF triplet states. Note that considering more SOF states in the first step of the calculation does not significantly improve the computed values, meaning that this formula explains most of the ZFS in axially distorted six-coordinate nickel(II) complexes.

Table 5. $A b$ initio $\Delta_{1}, \Delta_{2}$ and $D$ values and values of $D$ derived from the crystal-field expression $\left(D_{\mathrm{CF}}\right)$ obtained with $\zeta=648 \mathrm{~cm}^{-1}$ (all values are in $\mathrm{cm}^{-1}$ ) [Maurice, 2011].

\begin{tabular}{ccccc}
\hline$\tau_{a x}$ & $\Delta_{1}$ & $\Delta_{2}$ & $D$ & $D_{\text {CF }}$ \\
\hline 0.957 & 8382.4 & 9692.5 & -5.519 & -6.855 \\
0.971 & 8669.7 & 9559.4 & -3.568 & -4.564 \\
0.985 & 8964.0 & 9416.5 & -1.736 & -2.279 \\
1.000 & 9266.9 & 9266.9 & 0.000 & 0.000 \\
1.015 & 9576.9 & 9110.8 & 1.659 & 2.271 \\
1.029 & 9895.8 & 8951.8 & 3.259 & 4.530 \\
1.044 & 10224.0 & 8791.1 & 4.814 & 6.778 \\
\hline
\end{tabular}

In a similar way, one can introduce a rhombic distortion, i.e. an in-plane radial distortion. The crystal-field derivation leads to:

$$
D=-\frac{\zeta^{2}}{\Delta_{1}}+\frac{\zeta^{2}}{2 \Delta_{2}}+\frac{\zeta^{2}}{2 \Delta_{3}}
$$


and:

$$
E=-\frac{\zeta^{2}}{2 \Delta_{2}}+\frac{\zeta^{2}}{2 \Delta_{3}}
$$

where $\Delta_{1}$ correlates with the ${ }^{3} \mathrm{~B}_{1 \mathrm{~g}} \rightarrow{ }^{3} \mathrm{~B}_{2 \mathrm{~g}}$ energy difference in the $D_{4 \mathrm{~h}}$ symmetry point group, and where $\Delta_{2}$ and $\Delta_{3}$ both correlate with the energy of the ${ }^{3} \mathrm{~B}_{1 \mathrm{~g}} \rightarrow{ }^{3} \mathrm{E}_{\mathrm{g}}$ excitation in the same symmetry point group [Maurice, 2011]. Similarly, it can be shown that these formulae are supported by ab initio calculations [Maurice, 2011], corroborating that the outcomes of model complex studies which aim at establishing magneto-structural correlation can be safely explained by crystal-field models.

Before presenting the example of nearly-octahedral manganese(III) complexes, it is worth mentioning that such derivations are only valid close to ideal geometries of high symmetry, since it is assumed that the ground and excited SOF wave functions are either (i) not much affected by the distortion or (ii) affected in a way that can be easily modelled. In general, it is always advisable to perform ab initio calculations and to analyze the nature of the SOF wave functions of interest. For instance, large angular distortions usually result in a mixing of various configurations in such a complex way that pen-and-paper analytical derivations become cumbersome. Moreover, even if analytical derivations can be performed, if the resulting formulae are too complicated, they become pointless in practice for establishing or understanding magneto-structural correlations. In such cases, it is preferable to directly establish the correlations by means of ab initio calculations, as presented in section III.1.

Another interesting point concerns the role of the second coordination sphere. Although this effect is traditionally neglected in crystal-field models, it was shown by means of $a b$ initio calculations that the second coordination sphere can play a crucial role on the single-ion anisotropy in some particular cases [Maurice et al., 2011b; Bogdanov et al., 2013].

Nearly-octahedral manganese(III) complexes, corresponding to the $d^{4}$ configuration, are particularly interesting as they present non-intuitive ZFSs [Maurice et al., 2010a]. Although formulae which rationalize the ZFS of such systems are presented in the book of Abragam and Bleaney [Abragam and Bleaney, 1986], this case is often misinterpreted. Ab initio calculations showed that the external space cannot be restricted to quintet-spin SOF state components. Indeed three triplet-spin roots must also be included in the derivation to obtain accurate crystal-field formulae for this configuration [Maurice et al., 2010a]. The use of these newly derived expression lead to the energies of the ten spin components of the SOF ${ }^{5} \mathrm{E}$ state 
reported in Table 6. Note that the same wave functions as those obtained by Abragam and Bleaney with five quintet roots were obtained [Abragam and Bleaney, 1986]. The trend line $E_{\mathrm{CF}}=1.034 E_{\text {ab initio }}$ has an $R^{2}$ value of 0.9992 , meaning that (i) the crystal-field formulae presented in Table 6 are valid and (ii) a reduced effective SOC constant of $346 \mathrm{~cm}^{-1}$ has to be used in the crystal-field model to effectively account for covalency.

Table 6. Analytical crystal field expressions of the energies, ab initio and derived crystal field energies (in $\mathrm{cm}^{-1}$ ) [Maurice et al., 2010a]. For the computation of the crystal-field energies, the free-ion SOC of $352 \mathrm{~cm}^{-1}$ and $a b$ initio SOF excitation energies of $\Delta Q=13993 \mathrm{~cm}^{-1}$ and $\Delta T=11005 \mathrm{~cm}^{-1}$ have been used.

\begin{tabular}{cccc}
\hline Multiplicity & $E_{\text {analytical }}$ & $E_{\text {ab initio }}$ & $E_{\mathrm{CF}}$ \\
\hline Singlet & $2 \frac{4 \Delta Q \zeta^{2}+3 \Delta T \zeta^{2}}{8 \Delta Q \Delta T}$ & 16.813 & 17.900 \\
Triplet & $\frac{4 \Delta Q \zeta^{2}+3 \Delta T \zeta^{2}}{8 \Delta Q \Delta T}$ & 8.399 & 8.950 \\
& 0 & & \\
Doublet & & 0.000 & 0.000 \\
Triplet & $-\frac{4 \Delta Q \zeta^{2}+3 \Delta T \zeta^{2}}{8 \Delta Q \Delta T}$ & -8.890 & -8.950 \\
& $-2 \frac{4 \Delta Q \zeta^{2}+3 \Delta T \zeta^{2}}{8 \Delta Q \Delta T}$ & -17.775 & -17.900 \\
Singlet & & & \\
& & & \\
\hline
\end{tabular}

Similarly to the nickel(II) case, one can derive formulae for the $D$ and $E$ parameters of Mn(III) complexes which belong to the $D_{4 \mathrm{~h}}$ and $D_{2 \mathrm{~h}}$ symmetry point groups. In this case, analytical formulae can also be written down for the parameters appearing in the Stevens fourth-rank operators. However, as shown in [Maurice et al., 2010a], one can consider various approximations, i.e. neglecting (i) the Stevens fourth-rank terms (large distortions) and (ii) the degeneracy lift of the excited SOF multiplets of the octahedral situation $\left({ }^{3} \mathrm{~T}_{1 \mathrm{~g}}\right.$ and $\left.{ }^{5} \mathrm{~T}_{2 \mathrm{~g}}\right)$. For the former approximation, one should note that in the case of small distortions, the Stevens fourth-rank terms can be important as they are closely related to the near-degeneracy of the states that originate from the ${ }^{5} E_{g}$ of the octahedron [Maurice et al., 2010a]. In the $D_{2 \mathrm{~h}}$ symmetry point group, two configurations mix to form 
the two lowest SOF states [Abragam and Bleaney, 1986]. It is therefore necessary to introduce a mixing parameter $\delta$ to express these states as:

$$
\left|\phi_{1}\right\rangle=\cos \delta\left|Q_{1}\right\rangle+\sin \delta\left|Q_{2}\right\rangle
$$

and:

$$
\left|\phi_{2}\right\rangle=-\sin \delta\left|Q_{1}\right\rangle+\cos \delta\left|Q_{2}\right\rangle
$$

where, $Q_{1}$ and $Q_{2}$ are the two coupled configurations. This leads, after some pen-and-paper work, to [Maurice et al., 2010a]:

$$
D=\zeta^{2} \cos 2 \delta\left[\frac{3}{16 \Delta Q}+\frac{1}{4 \Delta T}\right]
$$

and:

$$
E=\zeta^{2}|\sin 2 \delta|\left[\frac{3}{16 \Delta Q}+\frac{1}{4 \Delta T}\right]
$$

where $E$ is defined positive by convention. The careful reader will notice that, as in the octahedral situation, the contribution of the triplet roots which were added in this derivation is proportional to the effect of the quintet roots that correlate with ${ }^{5} \mathrm{~T}_{2 \mathrm{~g}}$ in the octahedron. Finally, we end up with the same formula as the one reported in the book of Abragam and Bleaney [Abragam and Bleaney, 1986]:

$$
\frac{E}{|D|}=\frac{\sqrt{3}}{3}|\tan 2 \delta|
$$

To conclude, one should mention that the anisotropy parameters are not enlarged by distorting the first coordination sphere in this configuration, as illustrated by Figure 8 in the case of axial distortions. The ZFS of complexes belonging to this configuration is nonintuitive and the combined $a b$ initio and crystal field model study has proved to be enlightening for experimental applications. Indeed, in this configuration, it is pointless to synthesize complexes with large distortions to enlarge the ZFS parameters, which is 
notably consistent with the empirical fact that $d^{4}$ complexes typically have axial ZFS parameter values ranging between -5 and $5 \mathrm{~cm}^{-1}$ [Boča, 2004].

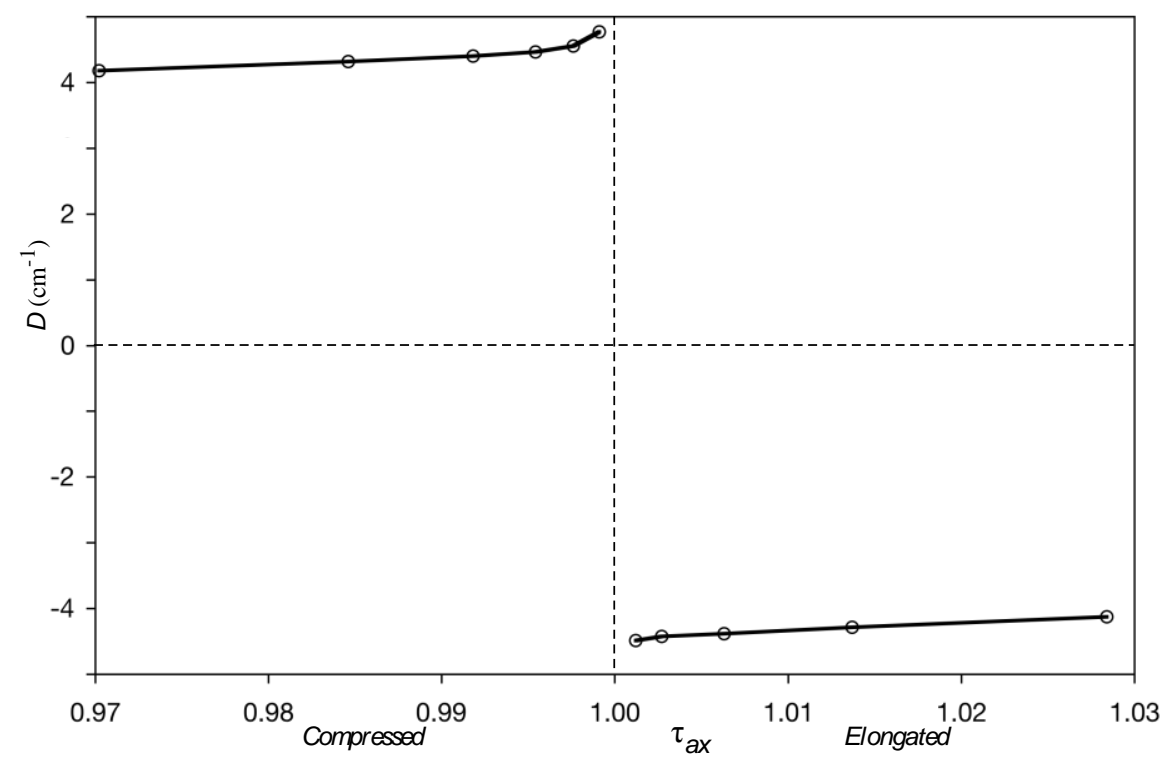

Figure 8. $A b$ initio $D$ parameter as a function of the axial distortion in model $D_{4 \mathrm{~h}}$ manganese(III) complexes [Maurice et al., 2010a].

\section{Conclusion}

In this chapter, we have shown that phenomenological Hamiltonians can be justified or even improved using the effective Hamiltonian theory; this creates a bridge between (supposedly) accurate $a b$ initio calculations and intuitive models. We have also exemplified how the crystal-field theory can be used to rationalize the nature and magnitude of ZFS. These tools allow us to take another step in the direction of the control of magnetic properties as they provide concrete understanding of how to increase the magnetic anisotropy. Magneto-structural correlations have also been established, which may help to design molecules with desired properties. To maximize single-ion anisotropies, researchers followed strategies such as exploring exotic coordination spheres (for instance pentacoordinate or heptacoordinate complexes), and even low-coordination spheres. Several efforts have been devoted to binuclear and polynuclear systems, more often concerning single-ion anisotropies, but also concerning giant-spin and multispin models. Consequently, substantial progress has been made in 
the understanding of the the magnitude and nature of the magnetic anisotropy in TM complexes with a wide range of ZFS parameter values over the last two decades.

Nevertheless, many aspects deserve further studies. For instance, the treatment of large systems is still problematic as (i) it is not clear which method can be used to obtain an optimal balance between accuracy and efficiency, and (ii) current models to describe polynuclear complexes may not be complete. Indeed, even in the case of mononuclear complexes, some cases are typically pathological, e.g. when heavy atom ligands are involved. In this situation, it is not clear yet if sum-over-states or c-SOCI approaches can be safely applied to the computation of the ZFS due to the truncation and stateaveraging errors [Maurice, 2011]. One may thus prefer to introduce the spin-dependent effects a priori, as done within $2 c$ frameworks. Also, some experimental data may have been incorrectly interpreted due to the use of inadequate models to fit the experimental outcomes of various techniques (magnetic susceptibility, magnetization, EPR, etc.). Therefore, there is clearly a need for more extensive studies and developments in the field of molecular magnetism. We hope that this chapter will motivate future work of this kind. 


\section{References}

[Abragam and Pryce, 1951] A. Abragam and M. H. L. Pryce, Proc. Roy. Soc. London Ser. A, 1951, 206, 164-172.

[Abragam and Bleaney, 1986] A. Abragam and B. Bleaney, Electron paramagnetic resonance of transition ions, Dover Publications, Dover, New York, 1986.

[Altshuler and Kozyrev, 1974] S. A. Altshuler and B. M. Kozyrev, Electron paramagnetic resonance in compounds of transition elements, John Wiley \& Sons, New York, 1974.

[Andersson et al., 1992] K. Andersson, P.-Å. Malmqvist and B. O. Roos, J. Chem. Phys., 1992, 96, 1218-1226.

[Angeli et al., 2001] C. Angeli, R. Cimiraglia, S. Evangelisti, T. Leininger and J.-P. Malrieu, J. Chem. Phys., 2001, 114, 10252-10264.

[Aquino and Rodriguez, 2005] F. Aquino and J. H. Rodriguez, J. Chem. Phys., 2005, 123, 204902.

[Atanasov et al. 2013] M. Atanasov, J. M. Zadrozny, J. R. Long and F. Neese, Chem. Sci., 2013, 4, 139-156.

[Bloch, 1958] C. Bloch, Nucl. Phys., 1958, 6, 329-347.

[Boča, 1999] R. Boča, Theoretical foundations of molecular magnetism, Elsevier, Amsterdam, 1999.

[Boča, 2004] R. Boča, Coord. Chem. Rev., 2004, 248, 757-815.

[Bogdanov et al., 2013] N. A. Bogdanov, R. Maurice, I. Rousochatsakis, J. van ben Brink and L. Hozoi, Phys. Rev. Lett., 2013, 110, 127206.

[Buckingham et al., 1982] A. D. Buckingham, P. Pyykko, J. B. Robert and L. Wiesenfeld, Mol. Phys., 1982, 46, 177-182.

[Caneschi et al., 1991] A. Caneschi, D. Gatteschi, R. Sessoli, A. L. Barra, L. C. Brunel and M. Guillot, J. Am. Chem. Soc., 1991, 113, 5873-5874.

[Chang et al., 1986] C. Chang, M. Pélissier and P. Durand, Phys. Scripta, 1986, 34, 394 404.

[Chibotaru and Ungur, 2012] L. F. Chibotaru and L. Ungur, J. Chem. Phys., 2012, 137, 064112.

[Costes et al., 2012] J.-P. Costes, R. Maurice and L. Vendier, Chem. Eur. J., 2012, 18, 40314040 .

[de Graaf and Broer, 2015] C. de Graaf and R. Broer, Magnetic interactions in molecules and solids, Springer, 2015 (ISBN: 978-3-319-22950-8). 
[de P. R. Moreira et al., 1999] I. de P. R. Moreira, F. Illas, C. J. Calzado, J. F. Sanz, J.-P. Malrieu, N. Ben Amor and D. Maynau, Phys. Rev. B, 1999, 59, 6593-6596.

[de P. R. Moreira and Illas, 2006] I. de P. R. Moreira and F. Illas, Phys. Chem. Chem. Phys., $2006,8,1645-1659$.

[des Cloizeaux, 1960] J. des Cloizeaux, Nucl. Phys., 1960, 20, 321-346.

[Dirac, 1929] P. A. M. Dirac, Proc. R. Soc. London Ser. A, 1929, 123, 714-733.

[Douglas and Kroll, 1976] N. Douglas and N. M. Kroll, Ann. Phys., 1976, 82, 89-155.

[Duboc et al., 2010] C. Duboc, D. Ganyushin, K. Sivalingam, M.-N. Collomb and F. Neese, J. Phys. Chem. A, 2010, 114, 10750-10758.

[Dzyaloshinskii, 1958] I. Dzyaloshinskii, J. Phys. Chem. Solids, 1958, 4, 241-255.

[Ederer and Spaldin, 2004] C. Ederer and N. A. Spaldin, Nat. Mater., 2004, 3, 849-851.

[Finley et al., 1998] J. Finley, P.-Å. Malmqvist, B. O. Roos and L. Serrano-Andrés, Chem. Phys. Lett., 1998, 288, 299-306.

[Ganyushin and Neese, 2006] D. Ganyushin and F. Neese, J. Chem. Phys., 2006, 125, 024103.

[Gatteschi and Sessoli, 2003] D. Gatteschi and R. Sessoli, Angew. Chem. Int. Ed., 2003, 42, $268-297$.

[Gomez-Coca et al., 2013] S. Gomes-Coca, E. Cremades, N. Aliaga-Alcalde and E. Ruiz, J. Am. Chem. Soc., 2013, 135, 7010-7018.

[Heisenberg, 1928] W. Heisenberg, Z. Phys., 1928, 49, 619-636.

[Herchel, 2007] R. Herchel, R. Boča, J. Krzystek, A. Ozarowski, M. Durán and J. van Slageren, J. Am. Chem. Soc., 2007, 129, 10306-10307.

[Hess, 1986] B. A. Hess, Phys. Rev. A, 1986, 33, 3742-3748.

[Hess et al., 1996] B. A. Hess, C. M. Marian, U. Wahlgren and O. Gropen, Chem. Phys. Lett., 1996, 251, 365-371.

[Iliaš and Saue, 2007] M. Iliaš and T. Saue, J. Chem. Phys., 2007, 126, 064102.

[Jansen and Hess, 1989] G. Jansen and B. A. Hess, Phys. Rev. A, 1989, 39, 6016-6017.

[Kahn, 1993] O. Kahn, Molecular Magnetism, VCH Publishers, 1993.

[Krzystek et al., 2004] J. Krzystek, S. A. Zvyagin, A. Ozarowski, A. T. Fiedler, T. C. Brunold and J. Telser, J. Am. Chem. Soc., 2004, 126, 2148-2155.

[Kubica et al., 2013] A. Kubica, J. Kowalewski, D. Kruk and M. Odelius, J. Chem. Phys., 2013, 138, 064304. 
[Llusar et al., 1996] R. Llusar, M. Casarrubios, Z. Barandiarán and L. Seijo, J. Chem. Phys., 1996, 105, 5321-5330.

[Malrieu et al., 2014] J.-P. Malrieu, R. Caballol, C. J. Calzado, C. de Graaf, and N. Guihéry, Chem. Rev., 2014, 114, 429-492.

[Maurice et al., 2009] R. Maurice, R. Bastardis, C. de Graaf, N. Suaud, T. Mallah and N. Guihéry, J. Chem. Theory Comput., 2009, 5, 2977-2989.

[Maurice et al., 2010a] R. Maurice, C. de Graaf and N. Guihéry, J. Chem. Phys., 2010, 133, 084307.

[Maurice et al., 2010b] R. Maurice, N. Guihéry, R. Bastardis and C. de Graaf, J. Chem. Theory Comput., 2010, 6, 55-65.

[Maurice et al., 2010c] R. Maurice, C. de Graaf and N. Guihéry, Phys. Rev. B, 2010, 81, 214427.

[Maurice et al., 2010d] R. Maurice, A. M. Pradipto, N. Guihéry, R. Broer and C. de Graaf, $J$. Chem. Theory Comput., 2010, 6, 3092-3101.

[Maurice, 2011] R. Maurice, PhD thesis, Université de Toulouse, 2011, available online at http://thesesups.ups-tlse.fr/1430.

[Maurice et al., 2011a] R. Maurice, K. Sivalingam, D. Ganyushin, N. Guihéry, C. de Graaf and F. Neese, Inorg. Chem., 2011, 50, 6229-6236.

[Maurice et al., 2011a] R. Maurice, L. Vendier and J.-P. Costes, Inorg. Chem., 2011, 50, 11075-11081.

[Maurice et al., 2012] R. Maurice, A.-M. Pradipto, C. de Graaf and R. Broer, Phys. Rev. B, $2012,86,024411$.

[Maurice et al., 2013a] R. Maurice, P. Verma, J. M. Zadrozny, S. Luo, J. Borycz, J. R. Long, D. G. Truhlar and L. Gagliardi, Inorg. Chem., 2013, 52, 9379-9389.

[Maurice et al., 2013b] R. Maurice, C. de Graaf and N. Guihéry, Phys. Chem. Chem. Phys., 2013, 15, 18784-18804.

[McWeeny and Mizuno, 1961] R. McWeeny, Y. Mizuno, Proc. R. Soc. London Ser. A, 1961, 259, 554-577.

[McWeeny, 1965] R. McWeeny, J. Chem. Phys., 1965, 42, 1717-1725.

[Miralles et al., 1993] Miralles, O. Castell, R. Caballol and J.-P. Malrieu, Chem. Phys., 1993, 172, 33-43.

[Moriya, 1960] T. Moriya, Phys. Rev., 1960, 120, 91-98.

[Moskvin, 2007] A. S. Moskvin, J. Exp. Theor. Phys., 2007, 104, 913-927.

[Neese and Solomon, 1998] F. Neese and E. I. Solomon, Inorg. Chem., 1998, 37, 6568-6582. 
[Neese, 2005] F. Neese, J. Chem. Phys., 2005, 122, 034107.

[Neese, 2006] F. Neese, J. Am. Chem. Soc., 2006, 128, 10213-10222.

[Neese, 2007] F. Neese, J. Chem. Phys., 2007, 127, 164112.

[Ostrovsky et al., 2002] S. M. Ostrovsky, R. Werner, D. A. Brown and W. Hasse, Chem. Phys. Lett., 2002, 353, 290-294.

[Ozarowski, 2008] A. Ozarowski, Inorg. Chem., 2008, 47, 9760-9762.

[Pederson and Khanna, 1999] M. R. Pederson and S. N. Khanna, Phys. Rev. B, 1999, 60, 9566-9572.

[Pradipto et al., 2012] A.-M. Pradipto, R. Maurice, N. Guihéry, C. de Graaf and R. Broer, Phys. Rev. B, 2012, 85, 014409.

[Rebilly et al., 2008] J.-N. Rebilly, G. Charron, R. Rivière, E. Guillot, A.-L. Barra, M. D. Serrano, J. van Slageren and T. Mallah, Chem. Eur. J., 2008, 14, 1169-1177.

[Retegan et al., 2014] M. Retegan, N. Cox, D. A. Pantazis and F. Neese, Inorg. Chem., 2014, 53, 11785-11793.

[Rogez et al., 2005] G. Rogez, J.-N. Rebilly, A.-L. Barra, L. Soraca, G. Blondin, N. Kirchner, M. Duran, J. van Slageren, S. Parsons, L. Ricard, A. Marvilliers and T. Mallah, Angew. Chem., Int. Ed., 2005, 44, 1876-1879.

[Roos et al., 1980] B. O. Roos, P. R. Taylor and P. E. M. Siegbahn, Chem. Phys., 1980, 48, $157-173$.

[Roos, 2005] B. O. Roos, in Theory and applications of computational chemistry: The first forty years, edited by C. E. Dykstra, G. Frenking, K. S. Kim and G. E. Scuseria, Elsevier: Amsterdam, 2005, chapter 25, 725-764.

[Roos and Malmqvist, 2004] B. O. Roos and P.-Å. Malmqvist, Phys. Chem. Chem. Phys., 2004, 6, 2919-2927.

[Ruamps et al., 2014] R. Ruamps, R. Maurice, C. de Graaf and N. Guihéry, Inorg. Chem., 2014, 53, 4508-4516.

[Ruamps et al., 2013a] R. Ruamps, L. J. Batchelor, R. Maurice, N. Gogoi, P. JiménezLozano, N. Guihéry, C. de Graaf, A.-L. Barra, J.-P. Sutter and T. Mallah, Chem. Eur. J., 2013, 19, 950-956.

[Ruamps et al., 2013b] R. Ruamps, R. Maurice, L. J. Batchelor, M. Boggio-Pasqua, R. Guillot, A.-L. Barra, J. Liu, E.-E. Bendeif, S. Pillet, S. Hill, T. Mallah and N. Guihéry, J. Am. Chem. Soc., 2013, 135, 3017-3026.

[Rudowicz and Chung, 2004] C. Rudowicz and C. Y. Chung, J. Phys.: Condens. Matter, 2004, 16, 1-23.

[Schmitt et al., 2011] S. Schmitt, P. Jost and C. van Wüllen, J. Chem. Phys., 2011, 134, 
194113.

[Spiegelmann and Malrieu, 1984a] F. Spiegelmann and J.-P Malrieu, J. Phys. B. Atom. Mol. Phys., 1984, 17, 1235-1257.

[Spiegelmann and Malrieu, 1984b] F. Spiegelmann and J.-P Malrieu, J. Phys. B. Atom. Mol. Phys., 1984, 17, 1259-1279.

[Stevens, 1952] K. W. H. Stevens, Proc. R. Soc. London Ser. A, 1952, 65, 209-215.

[Teichtel et al., 1983] C. Teichtel, M. Pélissier and F. Spiegelman, Chem. Phys., 1983, 81, $273-282$.

[Telser, 2006] J. Telser, J. Braz. Chem. Soc., 2006, 17, 1501-1515.

[Titiš and Boča, 2010] Titiš and Boča, Inorg. Chem., 2010, 49, 3971-3973.

[Titiš and Boča, 2011] Titiš and Boča, Inorg. Chem., 2011, 50, 11838-11845.

[van Lenthe et al., 1994] E. van Lenthe, E. J. Baerends and J. G. Snijders, J. Chem. Phys., 1994, 101, 9783-9792.

[van Lenthe et al., 1996] E. van Lenthe, J. G. Snijders and E. J. Baerends, J. Chem. Phys., 1996, 105, 6505-6516.

[Van Vleck, 1945] J. H. Van Vleck, Rev. Mod. Phys., 1945, 17, $27-47$.

[Zadrozny et al., 2013] J. M. Zadrozny, M. Atanasov, A. M. Bryan, C.-Y. Lin, B. K. Rekken, P. P. Power, F. Neese and J. R. Long, Chem. Sci., 2013, 4, 125-138.

[Zein and Neese, 2008] S. Zein and F. Neese, J. Phys. Chem. A, 2008, 112, 7976-7983. 